\title{
Transatlantica
}

Revue d'études américaines. American Studies Journal

1 | 2018

Slavery on Screen / American Women Writers Abroad: 1849-1976

\section{Le regard impossible}

Réflexions sur les présupposés et les mécanismes idéologiques des images de synthèse photoréalistes dans le blockbuster hollywoodien contemporain

Mathias Kusnierz

\section{(2) OpenEdition}

1 Journals

Édition électronique

URL : https://journals.openedition.org/transatlantica/11812

DOI : $10.4000 /$ transatlantica. 11812

ISSN : 1765-2766

Éditeur

Association française d'Etudes Américaines (AFEA)

Référence électronique

Mathias Kusnierz, «Le regard impossible », Transatlantica [En ligne], 1 | 2018, mis en ligne le 01 septembre 2019, consulté le 04 février 2023. URL : http://journals.openedition.org/transatlantica/ 11812 ; DOI : https://doi.org/10.4000/transatlantica.11812

Ce document a été généré automatiquement le 4 février 2023

\section{(c) $($ ) $(9)$}

Creative Commons - Attribution - Pas d'Utilisation Commerciale - Pas de Modification 4.0 International - CC BY-NC-ND 4.0

https://creativecommons.org/licenses/by-nc-nd/4.0/ 


\section{Le regard impossible}

Réflexions sur les présupposés et les mécanismes idéologiques des images de synthèse photoréalistes dans le blockbuster hollywoodien contemporain

\section{Mathias Kusnierz}

Dans son livre de 1966 intitulé Special Effects in Motion Pictures, Frank P. Clark expose, à destination des techniciens d'Hollywood, les différentes manières d'élaborer les effets spéciaux d'un film. Son inventaire s'étend du trucage le plus simple - un écran de fumée, par exemple (40-43) - aux effets spéciaux les plus élaborés, comme l'animation image par image (108-109) ou la construction de maquettes animées complexes $(89,146$, 152). Le livre de Clark se veut un exposé technique, sans parti pris, et appuyé sur une solide connaissance pratique du métier de concepteur d'effets spéciaux. Il fait ainsi fréquemment référence au travail des artisans les plus illustres d'Hollywood, comme Willis O'Brien, Gordon Jennings ou Ub Iwerks, mais aussi à des techniciens moins connus tels que Harry Barndollar, Lee Zavitz, John Hench, Joshua Meador ou Larry Hampton. Parmi les films qui nourrissent sa réflexion, l'auteur mentionne certains des derniers chevrons de l'industrie: Destination Moon (Destination... Lune!, Irving Pichel, Eagle-Lion, 1950), When Worlds Collide (Le Choc des mondes, Rudolph Maté, Paramount, 1951), War of the Worlds (La Guerre des mondes, Byron Haskin, Paramount, 1953), 20,000 Leagues Under the Sea (20.000 Lieues sous les mers, Richard Fleischer, Walt Disney Productions, 1954), The Lost World (Le Monde perdu, Irwin Allen, Twentieth Century Fox, 1960), The Devil at 4 O'Clock (Le Diable à 4 heures, Mervyn LeRoy, Columbia, 1961) ou encore The Birds (Les Oiseaux, Alfred Hitchcock, Universal, 1963)1.

Derrière le souci d'objectivité et d'actualité des informations exposées dans l'ouvrage, un important présupposé théorique se dessine cependant au fil des pages: le responsable des effets spéciaux ou le technicien doit toujours tâcher d'élaborer des effets qui donnent au spectateur le sentiment de la réalité, de sorte que le récit demeure crédible à tout moment. On peut formuler aisément l'envers de cet impératif : l'image doit faire illusion, et même, l'illusion de réalité que le film procure au spectateur ne doit jamais être interrompue. 
3 Il n'y a, dans ce présupposé, rien qui soit en désaccord avec les normes esthétiques et narratives du cinéma classique hollywoodien, fût-ce en 1966. La production hollywoodienne, même post-classique, demeure un cinéma de la continuité et de la transparence narratives, du "montage "invisible" et "psychologique" ", pour le dire avec Th. Schatz, qui se fonde le plus souvent sur des récits lisibles, efficaces, dramatiquement orientés et propres à engranger des profits. Grâce à la transparence narrative et à la fluidité du montage, le spectateur entretient avec le défilement des plans un rapport d'identification et d'adhésion: autrement dit, il regarde l'image de cinéma comme il adhère au réel (Bordwell, Staiger et Thompson 174-193). Les principes de transparence et de continuité narratives définissent - d'après les historiens - le paradigme classique et servent à assurer la stabilité du système de production (Lastra 200-201). Surtout, parce qu'ils garantissent l'efficacité narrative hollywoodienne, ils en fondent aussi la puissance idéologique, en assurant que le spectateur adhère au récit, donc aux discours des films, qui se confond bien souvent avec le discours de l'American way of life (Bordwell, Staiger et Thompson 3-41, 70-85, 341-352; Bidaud 20, 192-217). Ainsi, les mécanismes idéologiques des films permettent d'amoindrir les antagonismes du réel et de juguler l'expression des protestations d'ordre social ou politique. En cela, on peut dire que les films sont le lieu d'une intense production idéologique (Žižek, 1997 40-44).

4 Par "productivité idéologique», je ne veux pas dire que le cinéma hollywoodien manipule le spectateur, loin s'en faut. Les formes du cinéma hollywoodien mettent en jeu des idéologies, ou des systèmes de valeurs et de pensées, qui s'affrontent dialectiquement dans l'arène visuelle du récit : complotisme contre vérité d'État dans Transformers: Revenge of the Fallen (Transformers 2: La Revanche, Michael Bay, Paramount, 2009), écologie contre climatoscepticisme dans The Day After Tomorrow (Le Jour d'après, Roland Emmerich, 2004) et Geostorm (Dean Devlin, Warner Bros., 2017), anarchisme et désobéissance civile contre autoritarisme politique dans Mission: Impossible - Fallout (Christopher McQuarrie, 2018). Mais rares sont les films hollywoodiens qui imposent au spectateur un point de vue univoque sans que lui soit laissée la possibilité de le dépasser dialectiquement. Aussi sommes-nous toujours capables de distance critique face aux films: notre adhésion au récit ne détermine pas systématiquement notre pleine approbation à tel ou tel discours.

5 Par ailleurs, le caractère toujours plus spectaculaire des computer generated images et l'usage de dispositifs comme les projections stéréoscopiques (couramment appelées 3D) ont poussé plusieurs historiens et théoriciens à remettre en question le paradigme classique de la transparence, non pour s'en débarrasser mais pour l'amender (Paci, 2004 et 2006). Les travaux historiques sur la résurgence d'un cinéma des attractions dans la production contemporaine (Paci, 2003) et notamment dans les blockbusters hollywoodiens (Tomasovic) étudient ces nouveaux dispositifs visuels; ils réévaluent aussi la notion de transparence pour en élaborer une théorie plus complexe que celle proposée par Bordwell, Staiger et Thompson (174-193).

Dick Tomasovic montre que Jaws (Les Dents de la mer, Steven Spielberg, Universal, 1975) et Star Wars: Episode IV - A New Hope (Star Wars, épisode IV : Un nouvel espoir, George Lucas, Twentieth Century Fox, 1977), tout en signant le grand retour du récit à Hollywood (Berthomieu 29), ont aussi l'ambition de créer des «chocs visuels " à l'aide de séquences interminables, véritables morceaux de bravoure destinés à épuiser le regard du spectateur (Tomasovic 311-312, 319). Il montre aussi que James Cameron, Peter 
Jackson et Sam Raimi ont délaissé au cours de leur carrière un cinéma de la pure attraction au profit d'un intérêt renouvelé pour le récit, comme chez leurs aînés Lucas et Spielberg. Contrairement à ce qu'on observe chez ces derniers, ajoute-t-il, ce regain d'intérêt pour le récit ne vise pas à le revivifier mais bien à intégrer davantage les attractions de sorte qu'elles paraissent moins gratuites. Tomasovic montre ainsi les rapports complexes entre la transparence et le spectacle en mettant en question l'idée communément admise de la souveraineté de la première sur le second.

7 Je formule donc mes réflexions sur la productivité idéologique des images de synthèse photoréalistes en tenant compte de ces travaux : elle ne s'exerce pas sans partage ni de manière hégémonique. D'une part, la complexité séductrice des digital effects se fonde sur un plaisir conscient de l'effet qui accompagne l'immersion du spectateur sans l'asservir au spectacle ni l'en distancier absolument. D'autre part, la transparence narrative est régulièrement battue en brèche par des formes d'attraction spectaculaire; par conséquent la productivité idéologique est, très souvent, intermittente. Ce sont d'ailleurs ces intermittences qui ouvrent au spectateur les espaces de liberté herméneutique dont je parlerai plus loin.

8 Je voudrais tout de même nuancer la portée et l'ampleur des attractions étudiées dans les travaux que je viens de mentionner. Ils prennent parfois avec excès le contrepied du paradigme de la transparence, en posant que les attractions correspondent nécessairement à une "rupture narrative» et à «un rappel de son statut au spectateur $"$.

Si l'adresse au spectateur ne repose guère sur le regard à la caméra des acteurs, les mouvements d'appareil hyperboliques produisent une rupture narrative et révèlent au spectateur son statut. Sans oublier, parmi bien d'autres éléments, toute une série de plans qui fonctionnent par allusion (la scène d'amputation des tentacules d'Octopus, un clin d'œil à ses premiers films que Raimi adresse à ses spectateurs fidèles) et le recours à des gros plans burlesques (l'insert sur l'araignée qui mord Peter dans Spider-Man, un pur moment visuel, autonome, frappant et comique) ${ }^{3}$. (Tomasovic 316-317)

Les excès spectaculaires ne défont pourtant pas nécessairement la cohérence narrative, ce qu'a montré Scott Bukatman (75) en revenant sur les thèses célèbres de Laura Mulvey (1975) sur le plaisir visuel. Tomasovic semble justement ignorer que les « vues attentatoires" (Belloï 77-159) et la suppression agressive de la distance que le spectateur entretient avec l'écran renforcent aussi, par l'immersion dans le récit, l'adhésion de son regard à ce qu'il voit. Les attractions font basculer l'image de cinéma dans une modalité performative : «ça fait vrai!», pense-t-on devant l'écran, et cette exclamation intérieure signifie en réalité «c'est vrai!». Les attractions sont dialectiques : en même temps qu'elle exhibent notre statut de spectateur et la place que nous occupons devant l'écran, elles nous plongent au cœur de l'action en nous y impliquant physiquement. Par ailleurs, Tomasovic semble présupposer que tous les spectateurs font preuve du même regard savant. Or, si le public ordinaire, et notamment les adolescents auxquels ces films sont destinés par les producteurs, voient bien le spectacle hollywoodien dans son ensemble et le détail de certains procédés, ils ne perçoivent pas tous avec un regard analytique la virtuosité de la caméra et les références érudites à l'univers de Spider-Man et aux films de Sam Raimi. Lesquelles peuvent d'ailleurs décupler le plaisir du récit, selon que le spectateur est plus ou moins amateur d'intertextualité et de mise en scène visible. 
10 Le souci de transparence et de continuité narratives - y compris quand il ménage un espace pour les attractions - explique, mais en partie seulement, l'exigence de réalisme au fondement des effets spéciaux et, plus tard, des images de synthèse. Ce réalisme a pour fonction de remplir l'une des attentes courantes des spectateurs de l'époque: rêver au sein d'un cadre le plus crédible possible. Le cinéma hollywoodien entretient un rapport lâche au réel; il propose des récits qui ne rompent pas les liens du spectateur avec la réalité mais qui l'en éloignent cependant. C'est la raison pour laquelle il préfère généralement les films qui lui proposent une évasion hors de son quotidien à ceux qui le ramènent à la réalité (Nacache 392-395, 402 ; Koppes et Black 281-285). Pourtant, le spectateur veut que les modes visuels de cette évasion restent les plus réalistes possibles - ou tout du moins les producteurs pensent que c'est là le souhait du public ainsi, il continue de plébisciter les films de science-fiction tournés avec de véritables acteurs et présentant des environnements photoréalistes, alors que de tels films pourraient aujourd'hui être intégralement conçus sur ordinateur (Williams 289). Je vois là une double contradiction structurelle des effets spéciaux et des images de synthèse tels qu'ils sont pratiqués à Hollywood: ils visent à davantage de réalisme - et ce phénomène n'a fait que s'accroître au cours de leur histoire - mais ils le font le plus souvent dans des films que les spectateurs américains appellent "escapist fare " ${ }^{4}$. Il s'agit là de longs-métrages qui relèvent de genres fondés sur le merveilleux, l'imagination ou l'évasion tels que le film fantastique, le film d'horreur ou le film de science-fiction (Worland 49-50). Il existe bien sûr à Hollywood des images de synthèse qui ne relèvent pas du photoréalisme mais elles demeurent minoritaires dans les blockbusters; je fais donc le choix de les écarter de mon champ de réflexion pour cet article. Je fais l'hypothèse que cette double contradiction structurelle des effets spéciaux et des images de synthèse à Hollywood procède de mécanismes idéologiques profondément ancrés dans l'industrie du cinéma américain et que ceux-ci dissimulent leur nature idéologique ainsi que celle des effets spéciaux.

11 Afin de clore ce préambule, je voudrais définir ce que j'entends par le mot " photoréalisme ». Le terme désigne d'abord un courant de l'histoire de l'art apparu à la toute fin des années 1960 et popularisé grâce à l'exposition 22 Realists du Whitney Museum de New York en 1970 (Monte 12-15). En réaction à l'action painting, à l'abstraction et au minimalisme, le photoréalisme vise à produire des toiles qui donnent au spectateur l'impression de se trouver face à une photographie (Meisel 12). Tout l'enjeu de cette pratique est qu'elle se propose de reproduire des photographies réelles par les moyens de la peinture tout en appareillant celle-ci par des outils techniques modernes (agrandissements photographiques, reproduction par grille, procédés photomécaniques). Le paradoxe est peu ou prou le même pour les images de synthèse, qui visent à reproduire une image du réel grâce aux technologies numériques modernes. Ce détour par l'histoire de l'art permet de saisir l'enjeu de ces pratiques : le mot désignera, dans mon propos, la postulation ou la capacité d'une image à passer pour une photographie, donc une image exacte du réel, alors même qu'elle est conçue par des moyens informatiques. En infographie, on parle de rendu photoréaliste lorsque les images de synthèse ne se laissent pas distinguer d'une photographie et s'intègrent de manière invisible à des prises de vues réelles. Cette invisibilité n'empêche pourtant pas le spectateur de les déceler : il sait spontanément qu'un engin spatial, un corps cybernétique ou un dinosaure gigantesque sont des images de synthèse, quand bien même ils semblent réels. J'étudierai dans cet article ces images de synthèse photoréalistes. 


\section{Analyser les mécanismes de la production idéologique} réel et l'illusion, et en examinant les usages discursifs des effets spéciaux et des images de synthèse dans un corpus de blockbusters américains des années 1990 à aujourd'hui, j'entends donc mener une réflexion théorique dans laquelle je tâcherai de comprendre pourquoi le caractère idéologique de l'illusion créée par les effets spéciaux et les images de synthèse photoréalistes reste obstinément dissimulé. Dans le même mouvement, je tenterai aussi de révéler cette dimension idéologique, en analysant ses mécanismes et ceux de sa dissimulation. Enfin, j'examinerai à l'aide de quelques cas précis la manière dont les formes critiques - même au sein du cinéma hollywoodien - s'entendent à briser les mécanismes de la production idéologique mis en jeu par les effets spéciaux et les images de synthèse.

La question de l'idéologie sera donc le centre de gravité de cet article. Je l'envisage dans sa double acception, contemporaine et marxiste. À un premier niveau et dans son sens courant, l'idéologie est l'ensemble des valeurs et des idées qui structurent une société donnée; à un second niveau, dans son acception marxiste qui prolonge la première, l'idéologie est le système de représentations qui se prétend neutre alors qu'il fonde la structure de domination qui a cours dans une société donnée. Elle est la fausse conscience qui éloigne le prolétaire de la lutte sociale; elle "représente le rapport imaginaire des individus à leurs conditions réelles d'existence » (Althusser 101), c'est-àdire qu'elle leurre les travailleurs, de manière à ce qu'ils acceptent ces conditions comme immuables et justes. Or, dans le cinéma hollywoodien, ces deux acceptions travaillent de concert (alors qu'elles ne se recoupent pas nécessairement hors de la sphère cinématographique) : les films sont porteurs d'idéologèmes (Jameson 20) - des signes dont le signifiant masque la teneur idéologique du signifié - qui ne sont pas seulement des valeurs morales ou des idées, mais qui servent une structure de domination tout en se prétendant neutres.

Prenons un exemple. À travers son récit d'espionnage domestique, True Lies (James Cameron, Lightstorm Entertainment, 1994) ne cesse de rejouer les moments saillants de l'histoire militaire de l'Amérique: la guerre de Sécession, lorsque Arnold Schwarzenegger poursuit un terroriste à cheval, la Seconde Guerre mondiale lorsqu'il s'infiltre dans un manoir suisse gardé par des sentinelles aux allures d'officiers nazis, ou encore la guerre du Viêt Nam dans le plan où le jet Harrier décolle sur fond de soleil couchant, ce qui évoque, bien sûr, Apocalypse Now (Francis Ford Coppola, United Artists, 1979). Chacune de ces vignettes est coulée dans la geste individuelle d'un common man, en réalité héros national, qui impose avec son triomphe celui de l'Amérique. Mais il y a plus dans ces images que de simples valeurs historiques et morales. En faisant advenir la victoire historique et militaire, le récit entérine la domination patriarcale (c'est en sauvant son pays qu'Harry Tasker ramène son épouse sous le joug d'un mariage sans écarts extra-conjugaux) ainsi qu'une forme de ségrégation raciale où le terroriste est aussi le travailleur arabe victime de discrimination au travail. Quand Salim Abu Aziz, le terroriste en chef, se retrouve suspendu par les bretelles de son habit au nez d'un missile de l'avion de Tasker, ce dernier déclenche la mise à feu de l'engin d'un laconique « you're fired! ». Le spectateur américain entend bien sûr «t'es viré ! mais aussi, plus littéralement et eu égard aux images, «t'es explosé ! » ou «t'es cramé ! ». Le 
gros plan en travelling arrière sur le visage de Salim, grotesquement déformé par la vitesse du missile, humilie donc autant le travailleur immigré que le terroriste, tandis que le mouvement de la caméra accompagne (et accomplit) le meurtre du personnage. En véhiculant des valeurs d'ordre idéologique, les images travaillent donc aussi à renforcer la structure de la domination et à la rendre acceptable au spectateur ${ }^{5}$. J'ai étudié ailleurs la productivité idéologique de telles images (Kusnierz, 2017 ; 2019 ; 2020) et je voudrais ici me concentrer sur les soubassements idéologiques des images de synthèse photoréalistes, en tant qu'elles sont l'un des rouages de cette productivité.

\section{Les quatre paradoxes des effets spéciaux}

15 Avant d'entrer dans le vif de l'analyse, il me faut donner une précision terminologique. En français, on a tendance à utiliser l'expression «images de synthèse " comme un hyponyme de l'expression "effets spéciaux». Or, en anglais, les "special effects" désignent plutôt les effets analogiques, tandis que les «digital effects" désignent les effets conçus par ordinateur. Pour des raisons de clarté et de cohérence lexicale avec un certain nombre des sources que je cite, j'emploierai parfois l'expression «effets spéciaux », parfois " images de synthèse » ou " digital effects ». Ce sont ces digital effects que l'anglais appelle aussi «computer generated images » ou «CGI» - qui constituent l'objet principal de mon étude et qui présentent selon David Rodowick une ontologie distincte des effets spéciaux analogiques (Rodowick 163-174).

Les effets spéciaux et les images de synthèse sont des objets plastiques et visuels éminemment paradoxaux, et cette dimension contradictoire entre pour une bonne part dans la dissimulation de leur caractère idéologique. Non seulement leurs modes opératoires et figuratifs sont trop divers pour entrer dans une catégorie unique, mais leur déploiement dans les films et leur devenir sont eux-mêmes paradoxaux, comme nous allons le voir. Historiquement, les effets spéciaux sont la version moderne du «trucage ». Ce mot, écrit Réjane Hamus-Vallée (2004 5-6), apparait en 1872 sous la graphie « truquage »; il désigne la falsification d'objets et notamment d'objets d'art. Un peu plus tard, le Robert le définit comme suit : «Au spectacle, tout procédé d'illusion comportant l'emploi de trucs. » Le mot " truc » est quant à lui utilisé dès 1803 dans la prestidigitation et le théâtre. De fait, le syntagme "effets spéciaux » a une acception spécifiquement cinématographique, que ces effets soient mécaniques et réalisés sur le plateau ou visuels et réalisés en post-production. Dans les premiers ouvrages sur la question, le terme «illusion" apparaît fréquemment. Aussi Pierre Hémardinquer (5) donne-t-il cette définition, empruntée presque mot pour mot à Frank P. Clark :

Les « effets spéciaux » sont les techniques qu'ils [le metteur en scène et l'opérateur] utilisent pour assurer l'illusion visuelle et sonore des spectateurs, étendre la gamme des images et des sons au-delà de la réalité courante, créer des illusions d'action ou simuler des événements trop difficiles à filmer directement pour des raisons de sécurité, de possibilités pratiques ou de prix de revient. (Clark 5)

On peut déduire de ces considérations quatre paradoxes spécifiques aux effets spéciaux et qui en déterminent la nature idéologique. Le premier tient à ce qu'ils prétendent représenter le réel à partir d'artifices ; le deuxième est qu'ils relèvent explicitement de l'illusion tout en étant sous-tendus par une exigence de réalisme ; le troisième est que, très souvent, ils entendent représenter des objets impossibles tout en donnant à cet impossible les atours du réel. Plusieurs théoriciens l'ont remarqué. Pour Stephen Prince, les images de synthèse sont « réalistes sur le plan de la perception tout en étant 
non réelles sur le plan référentiel $»^{6}$. Pour Lev Manovich, elles "jouissent d'une crédibilité photographique parfaite bien qu'elles n'aient jamais été filmées ${ }^{7}$. On peut les résumer en un seul paradoxe, sous la forme d'une injonction contradictoire : l'effet spécial idéal est simultanément hypervisible et invisible; on doit le percevoir comme phénomène sans jamais en déceler la cause ; la plupart du temps on le voit et l'on sait qu'il s'agit d'un effet spécial mais simultanément on l'oublie pour continuer de croire au récit. Il serait donc cette "impression pure» dont parle Merleau-Ponty (10), sensation dont la cause nous échappe. Mais avec la cause, c'est également l'idéologie qui échappe à notre regard et notre vigilance, et qui s'impose alors d'autant mieux qu'elle passe inaperçue.

Le remake dit live de Ghost in the Shell (Rupert Sanders, DreamWorks, 2017) pousse très loin ce paradoxe. Adaptation en prises de vues réelles de l'anime de Mamoru Oshii, luimême adapté du manga de Masamune Shirow, le film ne cesse de faire évoluer son actrice de chair et d'os (Scarlett Johansson) dans des environnements simulés par informatique, tout en l'habillant d'une peau de synthèse elle-même conçue à l'aide d'outils numériques. Le photoréalisme des images de synthèse vient de leur complète homogénéité aux prises de vue réelles, au point que l'on oublie si ce sont les images informatiques qui sont parfaitement intégrées aux images live ou si ce n'est pas plutôt ces dernières qui sont intégrées aux images engendrées par l'ordinateur. Les logiciels de conception d'images de synthèse pour le cinéma comme Maya, Houdini ou Realflow simulent désormais le dispositif cinématographique (caméra virtuelle, profondeur de champ, cadrages, focales), de sorte que l'image filmique contemporaine devient un mixte de prises de vues réelles et d'images de synthèse où chacun de ces deux éléments est intégralement nécessaire à l'autre. Le paradoxe est d'autant plus visible dans les plans prélevés sur le film original, où ils étaient dessinés à la main : le saut dans le vide du Major en plongée zénithale, sa traversée au ralenti d'une baie vitrée, son combat à mains nues avec un malfaiteur alors qu'elle est invisible sont quelques-unes des scènes qui ont contribué à la renommée du film d'Oshii et qui se trouvent refaites, à l'identique ou presque, en prises de vues réelles et en images de synthèse. Tout se passe, dans le film de Rupert Sanders, comme s'il s'agissait d'incarner des personnages et des lieux d'abord immatériels, à l'aide d'outils qui mettent pourtant en doute l'incarnation et la matérialité des corps et des lieux. Et ce paradoxe se vérifie jusque dans l'ultime plan du film, où le Major saute dans le vide et devient invisible grâce à son camouflage thermo-optique. La technologie, qui fournit un corps glorieux au corps réel de Scarlett Johansson et qui donne vie aux environnements numériques du film, est aussi l'outil qui oblitère ce corps charnel de l'image.

La scène d'introduction nous montre la fabrication d'un corps cybernétique modélisé en images de synthèse, au gré de plans en pied enchaînés par des raccords sur le mouvement et sur le corps. Le spectateur observe la genèse du corps cybernétique, mais également, d'une certaine façon et au second degré, la fabrication de ce corps par l'ordinateur. Seul un gros plan en insert détaille le grain de la peau d'une main humaine et atteste la parfaite indistinction du corps humain et du corps cybernétique, bien que la coupe et la différence d'échelle tracent aussi, entre les deux corps, une invisible frontière. Le film ne cessera ensuite de montrer les états-limite de ce corps, ceux qui laissent apparaître la machine sous ou sur l'enveloppe humaine - à la manière dont Johansson, à la fin d'Under the Skin (Jonathan Glazer, Film4, 2013), ôtait sa peau humaine comme une combinaison et révélait sa nature extraterrestre. Ces états-limites 
sont autant de situations où le cinéma expose les modalités sous lesquelles il s'approprie le corps humain par la technologie et où l'on observe l'entrelacs parfait entre l'image filmée du corps humain et sa reproduction en images de synthèse (Momcilovic 75-84) ${ }^{8}$. Le corps du Major explose et révèle ses mécanismes métalliques après avoir bandé toute sa musculature en arrachant la tête du spider tank à la fin du film; ses tissus sont progressivement reconstitués après qu'il a échappé à une explosion, dans une scène qui rappelle $L a$ Leçon d'anatomie du Docteur Tulp (Rembrandt, 1632) (Momcilovic 72-74) et où le spectateur peut observer en transparence les différentes couches de cet être artificiel (ossature robotique, réseaux de veines et de circuits imprimés, derme et épiderme); Kuze ôte une plaque du visage du Major et révèle une étrange figure inhumaine où le vide mange les contours familiers du faciès humain ; à plusieurs reprises, le corps du Major disparaît sous les scintillations de son camouflage thermo-optique.

20 Ailleurs dans le film, les figures et les corps hybrides prolifèrent, et il n'est pas indifférent que l'espace urbain représenté dans Ghost in the Shell soit saturé d'apparitions holographiques qui, presque toutes, procèdent à un écart figuratif avec le corps humain, qu'elles le déforment, en restituent une aura vacillante et spectrale ou le représentent comme un assemblage de pixels (Lécole Solnychkine). Aussi l'image viset-elle toujours deux horizons contradictoires que je désignerai, s'il m'est permis, par deux néologismes. D'une part elle est halographique (contraction de "halo» et $d^{\prime}$ '«holographie») et pulvérise la silhouette humaine en un nuage de miroitement lumineux ; d'autre part elle est hallographique (contraction du grec " allos ", " autre ", et d'« holographie ») et déforme le corps humain de manière infinitésimale, par une série d'écarts inframinces qui le rendent autre (Duchamp 24). Dans les deux cas, la figure humaine est comme parasitée de l'intérieur par l'image numérique. Mais paradoxalement, c'est le surgissement de l'informatique depuis le cœur même de l'image filmique qui assure l'indistinction entre les prises de vue réelle et les images conçues sur ordinateur (Balides 315).

Dans ce cas particulier comme dans bien d'autres, les effets spéciaux (images de synthèse, pyrotechnie) sont à la fois invisibles et hypervisibles, comme le signalait déjà Clark : «De bons effets spéciaux se confondent avec la réalité sans que le public ne s'aperçoive immédiatement de l'illusion ${ }^{9}$. En tant qu'ils forment la matière même de la profusion visuelle, on ne voit qu'eux : ils sont le tout de l'image. Mais dans le même temps, ils se dissimulent en tant qu'artifices aux yeux du spectateur puisqu'ils sont entièrement intégrés aux prises de vues réelles et deviennent, dans l'écrin qu'elles forment, pareils à la réalité. Le photoréalisme tient donc à la facture même des images de synthèse, certes, mais surtout à la suture invisible entre les digital effects et les prises de vues réelles. Je développe plus loin les raisons pour lesquelles cette suture invisible revêt une fonction idéologique.

Il existe enfin un quatrième paradoxe, qui a trait au devenir historique des effets spéciaux. Inexorablement, leur nouveauté s'émousse, à cause des mécanismes d'habituation et d'automatisme qui tendent à rendre conventionnelles nos expériences ou, précise Chklovski, à nous rendre moins conscients du vif de l'expérience. Non sans dérision, le théoricien russe écrit : "l'automatisation dévore les objets, les habits, les meubles, votre épouse et la peur de la guerre » (22). La déflation de la nouveauté est donc, dans un premier temps du moins, implacable et irréversible, et elle détermine à la fois une "surenchère visuelle » des effets spéciaux et leur banalisation consécutive 
(Hamus-Vallée, 2004 8). Par conséquent l'histoire des effets spéciaux est celle de leur perfectionnement incessant, à travers lequel se réalise progressivement l'hégémonie du photoréalisme, elle-même liée à "l'hégémonie du numérique " qu'évoque Thibaut Garcia (48). En s'imposant de cette manière, à travers la marche même de la technologie vers le progrès, qu'Hollywood présente presque toujours comme irrésistible, le réalisme apparaît naturalisé, comme s'il était le destin de l'image cinématographique (Dubray 206; Lastra 210-211; Balides 315-320) ${ }^{10}$. De tels discours montrent combien l'exigence de photoréalisme qui fonde les effets spéciaux et les images de synthèse est informée idéologiquement et fondée sur des mécanismes qui relèvent de l'idéologie.

\section{Idéologie du photoréalisme}

Notre hypothèse est que la dimension de part en part contradictoire des effets spéciaux vaut pour indice du caractère idéologique de leur photoréalisme ; elle suggère que cette exigence n'est pas seulement due aux normes narratives du cinéma classique hollywoodien mais qu'elle endosse une valeur idéologique - que je préciserai plus loin le plus souvent dissimulée par l'excès de spectacle, la dépense d'énergie (cinétique, graphique, pyrotechnique) et la profusion visuelle des effets spéciaux et des images de synthèse. Le livre de Frank P. Clark contient ainsi une mention sur sa page de garde : "Avec l'accord du comité de conseil sur les effets spéciaux cinématographiques de la Société des Ingénieurs du Film et de la Télévision» (3). Un tel sceau d'approbation certifie que l'ouvrage est bien conforme au programme théorique et esthétique implicite défini par les instances de régulation d'Hollywood. Les réflexions de James Lastra sur l'évolution des technologies à Hollywood montrent que le cœur du problème est de nature idéologique. D'après lui, c'est le principe même du réalisme qui guide la production et l'adoption des normes:

La presse scientifique et industrielle regorge d'articles où les professionnels défendent avec passion divers modèles esthétiques, mais dans quel but? Quel rôle joue l'articulation entre standards esthétiques et normes? Celle-ci est loin d'être secondaire : les techniciens semblent souvent obnubilés par la volonté de débattre de leurs positions au sujet de questions telles que l'illusion mimétique, la netteté de l'image, les brevets techniques et la validité des procédés employés, et ils justifient presque systématiquement les standards qu'ils élisent par un discours sur la transparence réaliste ${ }^{11}$.

Par conséquent, l'évolution des technologies à Hollywood (et avec elles, bien sûr, celle des effets spéciaux) est le processus qui permet au réalisme de s'imposer de manière hégémonique, puis d'asseoir et de renforcer toujours plus cette hégémonie. Il en va ainsi des effets spéciaux comme des techniques de tournage plus traditionnelles : ils sont fondés sur une exigence première de réalisme et de transparence, bien que dans certains cas ils doivent exhiber leur dimension spectaculaire. Réjane Hamus-Vallée (2004 3) relève ainsi cette double postulation des effets spéciaux: selon le type de production, il faut que les effets spéciaux soient spectaculaires ou au contraire invisibles.

Aujourd'hui, quasiment tous les films font appel aux effets spéciaux: la pluie à volonté, des blessures sans conséquence, une affiche disgracieuse gommée d'un coup de palette graphique... Le numérique a facilité les petites retouches de l'image qui, par contrecoup, deviennent totalement banales. Ces effets invisibles semblent à l'opposé des effets spectaculaires mis en avant dans l'étiquette "films à effets 
spéciaux ». Ces derniers relèvent de la monstration, si ce n'est bien souvent du monstre : ils sont faits pour être vus, alors que les effets invisibles, justement, ne doivent pas se voir, ni même se sentir.

Dans l'ensemble de la production cinématographique contemporaine, seul le cinéma hollywoodien atteint à ce degré de photoréalisme. On pourrait croire que cet état de fait est simplement dû aux budgets démesurés dont dispose l'industrie du cinéma américain et, certes, on n'aurait pas tout à fait tort. Mais c'est que les sommes colossales qu'injecte Hollywood dans son cinéma constituent déjà en soi un phénomène idéologique (Bidaud 51-86). De fait, cette différence quant au degré de photoréalisme n'est-elle pas plutôt due à de profondes différences culturelles? Les trois épisodes de Détective Dee (Tsui Hark, Huayi Brothers Media, 2010, 2013 et 2018), par exemple, ont tout du blockbuster tel qu'un studio américain pourrait le concevoir - à ceci près que leurs budgets sont bien inférieurs (respectivement 13, 24 et 25 millions de dollars). Leur option esthétique dominante n'est pourtant pas celle du photoréalisme. Bien au contraire, dans la continuité de l'opéra, ces films ont recours à une palette de couleurs chatoyantes et des éclairages caravagesques; ils relèvent bien plus du pittoresque que de l'entreprise réaliste. À Hollywood, le cinéma d'heroic fantasy contemporain, comme la trilogie du Seigneur des anneaux (Peter Jackson, New Line Cinema, 2001-2003), insiste plutôt sur l'effet de réel, où «le "réel concret" devient la justification suffisante du dire ", et qui " procède de l'intention d'altérer la nature tripartite du signe pour faire de la notation la pure rencontre d'un objet et de son expression" (Barthes 87, 89), comme s'il y avait bien une contiguiité entre le réel et sa représentation filmique ou textuelle.

Il me faut bien sûr ajouter à ces considérations que le photoréalisme est autant le fruit d'une intuition du spectateur (il semble que certaines images paraissent à notre œil plus réalistes que d'autres) que d'une construction culturelle, qui nous accoutume à percevoir certaines images comme réalistes. La manière dont la photographie du cinéma américain évolue décennie après décennie me semble tout à fait révélatrice de cette construction culturelle. Les pastels des années 1980 ont fait place à des contrastes plus nets de bleus et de rouges dans les années 1990. Dans les années 2000, la mode a plutôt été à la désaturation des couleurs : ainsi de films comme Letters from Iwo Jima (Lettres d'Iwo Jima, Clint Eastwood, Warner Bros., 2006) ou The Bourne Supremacy (La Mort dans la peau, Paul Greengrass, Universal, 2004), dont la photographie est dominée par les nuances de gris. Mais pour le spectateur de chaque décennie, les films qui lui sont immédiatement contemporains sont toujours ceux dont l'image lui semble la plus 
photoréaliste possible, parce qu'en l'éloignant provisoirement de ses habitudes perceptives, ils semblent lui restituer le réel à l'état brut.

\section{Les trois qualités idéologiques des images de synthèse}

\section{L'excès visuel et sonore comme dissimulation de l'idéologie et de ses mécanismes}

Mon corpus d'étude est constitué, pour l'essentiel, de blockbusters américains sortis entre 1990 et aujourd'hui. Dans son bornage temporel, il correspond à l'apparition des digital effects photoréalistes dans des films à grand spectacle, dont la formule est pourtant ancienne à Hollywood: que l'on pense à The Ten Commandments (Les Dix Commandements, Cecil B. DeMille, Paramount, 1956) ou encore à Cleopatra (Cléopâtre, Joseph L. Mankiewicz, Twentieth Century Fox, 1963) et plus près de nous à Jaws et Star Wars. Au cours de la dernière décennie du $\mathrm{xx}^{\mathrm{e}}$ siècle, grâce aux progrès technologiques en matière d'effets spéciaux, d'images de synthèse et de procédés sonores et visuels immersifs, le cinéma hollywoodien devient capable de produire de l'illusion à un niveau jusqu'alors inégalé : aussi des films comme Terminator 2: Judgement Day (Terminator 2: Le Jugement dernier, James Cameron, TriStar Pictures, 1991), Jurassic Park (Steven Spielberg, Universal, 1993) et plus tardivement Avatar (James Cameron, Twentieth Century Fox, 2009) ont-ils fait date dans l'histoire des représentations à Hollywood (Lemieux 7-12) ${ }^{12}$. De fait, un véritable pallier qualitatif en termes de photoréalisme des images de synthèse semble franchi avec Terminator 2: Judgement Day (Cotta Vaz 201). Angela Ndalianis le formule en ces termes :

Notre époque se caractérise par ses transformations radicales, similaires à celles que le XVII ${ }^{\mathrm{e}}$ siècle a connues : les progrès effectués dans le domaine des technologies optiques ont permis de rendre visible ce qui était auparavant invisible et de construire des réalités alternatives et des environnements numériques capables d'offrir des expériences sensorielles réalistes ${ }^{13}$.

En qualifiant de "néo-baroque» le cinéma spectaculaire contemporain, Angela Ndalianis signale que l'une de ses premières vocations est la création d'illusions. Dans les trois films cités ci-dessus, la dépense spectaculaire est indissolublement liée à la production de faux-semblants visuels et définit pour l'image de cinéma une « folie du voir ", selon l'expression de Christine Buci-Glucksmann (20-26), soit une profusion visuelle excédant le langage qui voudrait s'en saisir et qui déborde les cadres de la représentation. Dans Transformers: Revenge of the Fallen, la séquence inaugurale, située en Chine, nous montre une opération militaire de grande ampleur destinée à capturer un Decepticon, une machine de guerre polymorphe d'origine extraterrestre. Les robots en images de synthèse, argument promotionnel majeur du film, déploient à l'écran des formes mi-mécaniques mi-organiques en métamorphose constante et figurent des objets que le langage ne saurait nommer, dans " un visible qui ne fait pas voir " (Rancière 137). Ce phénomène que signale Rancière rejoint l'un des reproches récurrents de la critique : dans ce film au coût faramineux, le spectateur ne distingue jamais nettement les effets spéciaux et les métamorphoses des robots. La profusion visuelle déroute et empêche le regard, dans un régime de vision très singulier où les digital effects se signalent tout en se dérobant. 
Le robot ennemi ressemble ici à une structure métallique montée sur de gigantesques roues, amalgame de monster truck et de plateforme pétrolière. Autour de lui, la caméra virevolte en d'incessants travellings circulaires et tremble lorsqu'elle est portée à l'épaule ; les explosions se multiplient ainsi que les forts contrastes de lumière, les faux raccords et les plans très courts. La facture même du film correspond bien à une forme de "folie du voir ", tandis que les éléments profilmiques relèvent quant à eux de la dépense pyrotechnique. On pourrait croire cette surabondance visuelle dépourvue de valeur idéologique parce qu'elle court-circuite le discours et la pensée pour ramener le spectateur à une pure passivité sensorielle, en fondant son corps dans celui du film ainsi que l'ont montré Merleau-Ponty (173-175) et Sobchack (203-248). Si la dépense revêt ici une valeur idéologique, c'est parce qu'elle dessine une équivalence entre l'excès pyrotechnique et la figuration de la puissance de feu de l'armée américaine. La profusion et l'excès revêtent donc une valeur idéologique d'autant plus forte qu'ils la dissimulent sous un tourbillon de stimuli sensoriels, supposés étrangers a priori à la signification. Ils sont donc l'idéologie par excellence, tant il est vrai que celle-ci se manifeste à son maximum d'intensité dans les phénomènes ou les objets les plus utilitaires ou triviaux, là où elle semble n'avoir pas droit de cité, comme l'ont montré respectivement Panofsky (183) et Žižek (1997 3-5) à propos de la calandre Rolls-Royce et de la forme propre aux cabinets de toilette des différentes nations d'Europe. Mais plus encore, le réalisme même de la représentation de cette créature impossible qu'est le Decepticon accrédite par la bande la probabilité d'incidents diplomatiques et militaires entre la Chine et les États-Unis et entretient, chez le spectateur, la peur des grandes puissances étrangères. Lorsque l'impossible semble réel, l'improbable se confond avec le vrai. Les représentations construites par les effets spéciaux photoréalistes forment donc, dans le contexte de leur scénarisation, des discours invisibles qui contribuent à remodeler, chez le spectateur, les frontières du fait attesté, du probable et du possible.

\section{Oblitérer les antagonismes du réel}

D’après Angela Ndalianis, le début des années 1990 est considéré comme le moment de l'histoire du cinéma hollywoodien où les images de synthèse parviennent à une première maturité (151). Dans ces images, la solution de continuité entre la représentation et l'objet, ou la suture entre l'image et le réel, est devenue presque invisible. Par conséquent, le cinéma accède pour la première fois à la possibilité d'une illusion sans interruption, même lorsqu'il représente des entités ou des phénomènes rigoureusement impossibles. L'androïde en métal liquide de Terminator 2: Judgement Day ou le tyrannosaure de Jurassic Park surgissant, grandeur nature ou presque, sur un écran panoramique, sont emblématiques du fossé entre réel et représentation que les effets spéciaux contemporains semblent avoir comblé - bien que la plupart des scènes du film de Spielberg mêlent effets numériques et analogiques. Les images de synthèse méritent enfin leur nom : elles sont devenues capables de synthétiser le réel à partir d'algorithmes.

Elles accèdent dès lors à la possibilité de représenter l'impossible, excédant ainsi toute les limites de la représentation pour basculer dans l'anti-représentation (Rancière 134-139). Dans le cas précis que je m'apprête à évoquer, l'anti-représentation correspond au déploiement, à l'écran, d'une situation impensable et impossible mais bel et bien accessible aux sens du spectateur. Slavoj Žižek (1997 16-18) nomme « regard impossible » cette capacité des récits ou des films à figurer ce dont le sujet ne saurait 
être le témoin, comme le moment de sa conception ou de son enterrement. Le regard impossible relève donc de la dystopie ou du récit fantasmatique. Or, poursuit Žižek, il est l'un des moyens privilégiés des appareils de contrôle idéologique: la «boucle temporelle » que forment à la fois le regard impossible et le récit fantasmatique qu'il sous-tend court-circuite la lucidité critique et la raison, et impose au spectateur d'accueillir discours et images sans distance ni vigilance. De fait, un film qui met en jeu des situations totalement dystopiques ou un regard impossible serait paradoxalement - davantage perméable à l'idéologie qu'un film visant à représenter concrètement des situations réelles ou des problèmes politiques ou sociaux. N'est-ce pas le cas d'Avatar, dont la fable écologique parvient à faire oublier combien le cinéma hollywoodien est mû par des intérêts financiers, industriels et politiques absolument contraires à ceux que le film prétend défendre (Žižek, 2010 66-69) ? De fait, l'idéologie que véhiculent les films semble toujours plus acceptable ou moins violente quand elle est déréalisée et perd de son poids concret. C'est que la fantasmagorie (dans laquelle on fait entrer sans peine images de synthèse et effets spéciaux) gomme les antagonismes du réel sous les atours du fonctionnalisme (l'image sert un récit) et les rend tolérables (Žižek, 1997 7-8, 40-44).

La fin de $x X x:$ Return of Xander Cage ( $x$ Xx: Reactivated, D. J. Caruso, Paramount, 2017) met littéralement en jeu ce regard impossible. Tué au début du film par la chute intempestive d'un satellite (sic), Augustus Gibbons refait surface au cours de l'épilogue dans l'église où l'on célèbre ses funérailles, accompagné du footballeur Neymar, sa nouvelle recrue pour le programme xXx. Accordons notre crédit à ce que dit, littéralement, le scénario : Gibbons et Neymar sont bel et bien morts au début du film et Gibbons assiste pour de bon à son enterrement. Le travail du film consiste ici à assurer la continuité logique de ces deux moments contradictoires. Aucun des membres de l'assemblée, à l'exception de Cage, ne verra d'ailleurs Gibbons. La caméra s'élève lentement, au cours d'un pano-travelling, vers le balcon de l'église où se trouve Cage, rejoint bientôt par Gibbons. Le mouvement de l'appareil, fluide, fait passer le regard du spectateur d'un espace à l'autre sans solution de continuité et les fait coexister alors même qu'ils devraient s'exclure (l'un contient Gibbons vivant, l'autre le contient mort : le cinéma est pareil à l'expérience de Schrödinger). Nous assistons donc à une situation qui défie les lois de la géométrie euclidienne mais que le cinéma rend possible par la grâce de son travail de fiction et d'imagination (mise en mouvement des images, montage). L'intérieur de l'église est, quant à lui, pareil à une salle de cinéma, avec sa fosse, son balcon et son estrade sur laquelle se trouve le cercueil, objet de tous les regards, et qui figure par métonymie le film lui-même, puisque celui-ci a pour origine la mort de Gibbons. De sorte que nous sommes bien, dans cette scène, coupés du réel, puisqu'elle représente métaphoriquement un lieu destiné à éloigner le spectateur de la réalité. En nous plongeant au cœur de cette situation impossible (un même personnage est, dans un même lieu, simultanément mort et vivant, débout sur un balcon et couché dans un cercueil), le film neutralise notre vigilance critique et nous prépare à accepter des discours que nous n'aurions pas nécessairement acceptés davantage que le retour de Gibbons devant sa propre dépouille. Au premier chef, le film nous prépare à voir sa suite en salles (donc à consommer d'autres films), puisque Gibbons demande à Cage de reprendre du service en ces termes : «Let me simplify it for you: kick some ass, get the girl, and try to look dope while you're doing it $\aleph^{14}$. Le triple impératif qu'il formule ici est un programme spectaculaire (action, érotisme, attitude des corps à l'écran) autant qu'idéologique, puisqu'il définit à l'usage du spectateur le partage entre bien et mal, 
entre masculin et féminin, ainsi qu'entre le style et l'absence de style. Dans la bandeannonce, cette réplique est superposée, en voice-over, aux images de l'ultime cascade du film: Vin Diesel s'éjecte d'un avion qui s'apprête à percuter un satellite. La caméra effectue un travelling arrière rapide qui fait défiler sous nos yeux tout l'intérieur de l'appareil, avant de suivre l'acteur en chute libre. Ici, seul Diesel est filmé sur fond vert, le reste de l'habitacle étant modélisé en images de synthèse, lesquelles simulent par ailleurs le dispositif cinématographique. Le mixte entre le corps réel de l'acteur et la simulation, par ordinateur, de l'appareillage de tournage, a là encore pour fonction de neutraliser notre vigilance critique, et nous prépare à accepter l'impératif pour le moins réactionnaire de Gibbons.

\section{Assigner le regard du spectateur}

La maturité mimétique des images conçues par ordinateur et des effets spéciaux croît sans cesse, et avec elle leur capacité à lisser les antagonismes du réel ${ }^{15}$. En corollaire, la spécularité du cinéma hollywoodien contemporain accède à un niveau quasi systématique, ce qui accroît d'autant la puissance d'illusion des images. À la limite, tout effet spécial est réflexif parce qu'il prétend au réel alors qu'il montre l'impossible, et interroge de cette manière la représentation. Dans cet écart contradictoire entre le réel et ce qui n'existe pas, une pensée de l'image s'ouvre qui se développe dans et par l'image. Le régime spéculaire du blockbuster contemporain confirme donc la grille analytique néo-baroque d'Angela Ndalianis. La question est, dans un second temps, de savoir ce que les films font de cette réflexivité : l'emploient-ils à consolider la puissance idéologique des films ou plutôt à la contrer? Dans Total Recall (Paul Verhoeven, TriStar Pictures, 1990), l'hologramme qu'utilise Doug Quaid pour tromper ses ennemis au cœur des ruines de la civilisation extraterrestre sur Mars vaut pour métonymie du film entier, où chaque image pourrait bien n'être que le fruit des fantasmes du héros (Ledoux 40-47). Inception (Christopher Nolan, Warner Bros., 2010) explore l'analogie déjà ancienne entre le rêve, la pensée et l'image filmique (Deleuze 25-32) : Ariane, "l'architecte", conçoit mentalement, au cours d'un rêve, le décor de l'opération d'« inception» (implanter une idée dans l'inconscient d'une cible, à son insu), une opération que les personnages doivent mener à bien dans le film. Ariane déforme et transforme ce décor à sa guise comme si elle disposait d'une palette graphique. L'identité entre l'image filmique et la pensée est totale puisque les métamorphoses qu'imagine Ariane sont avant tout celles que conçoivent les techniciens en charge des effets spéciaux du film. Et, alors que Total Recall peut être lu comme le récit marxiste de l'émancipation d'un ouvrier, Inception nous montre un individu qui reste prisonnier de ses fantasmes. Dans Edge of Tomorrow (Doug Liman, Warner Bros., 2014) enfin, la répétition de la même scène de guerre en une boucle temporelle sans fin ôte toute réalité aux événements représentés. Le héros meurt chaque fois à la fin de la scène mais se réveille dans le passé et revit les mêmes événements comme dans un jeu vidéo, de sorte que l'image filmique est implicitement désignée comme une illusion. Strange Days (Kathryn Bigelow, Lightstorm Entertainment, 1995) ou Minority Report (Steven Spielberg, Twentieth Century Fox, 2002) se prêtent eux aussi à de telles analyses, comme si l'industrie trouvait dans la spécularité du blockbuster un espace idéal dans lequel interroger ses propres modes de représentation, de narration et de diffusion. La prolifération des effets spéciaux dans ces cinq films semble en surdéterminer la spécularité, ce qu'Alain Boillat signalait déjà dans Cinéma, machine à mondes (51-56). 
Laquelle, nous allons le voir, peut aussi bien accroître l'illusion mimétique que la renverser, neutraliser la vigilance critique du spectateur aussi bien que l'aiguiser.

Les films que je viens d'évoquer figurent tous le dispositif cinématographique de façon détournée (Bolter et Grusin 53-84,147-158). Cette réflexion du cinéma américain sur son médium tend à laisser supposer qu'il a pleinement conscience de ce que la diffusion idéologique est avant tout une question de support, selon le mot célèbre de McLuhan (7-23) : « le médium est le message ». Dans Minority Report, l'appareil de prévision des crimes que Tom Cruise manipule au début du film relève à la fois de l'écran tactile et de la table de montage, tandis que le bassin où reposent les precogs évoque le caractère immersif de la salle de cinéma et le bain amniotique des images. Dans Strange Days, le SQUID est un cinématographe directement relié aux neurones des personnages; à travers lui, le film explore la nature idéologique des supports de diffusion d'images (et réciproquement la dimension matérielle de l'idéologie) en un récit qui dissout la résistance politique dans l'illusion, alors qu'à la toute fin du film la révolution avorte (Bolter et Grusin 3-15, 166). En représentant cette insurrection mort-née, le film construit deux discours contradictoires. Il montre la faillite de la résistance politique à l'œuvre, de manière à produire chez le spectateur un rapport critique aux images. Mais en en montrant l'échec, il met également fin à cette résistance politique dont il sanctionne l'impuissance au niveau du récit. Si le film détermine le regard du spectateur, il lui conserve donc également la possibilité d'une interprétation libre du récit par le jeu même de ces discours contradictoires, et le public garde toujours le choix d'y voir ce qu'il y cherche (Nacache 402). Le film est comme l'« étrange toupie » dont parle Sartre (48) au sujet du texte littéraire : sous l'œil du spectateur, il fait défiler l'orbe complet de ses significations contradictoires.

36 La tendance du cinéma hollywoodien contemporain à penser ses propres modes de production d'images apparaît donc profondément liée à la genèse de l'illusion. Ce rapport existe déjà à la période baroque, où la manipulation des images est indissociable d'un besoin de penser celle-ci, comme pour donner un cadre rigoureux aux irrégularités plastiques et autres déformations (Baltrušaitis, 1983 6-7, 37-40; 1984 5, 15-21, 211-220 ; Prigent 135). Mais il s'impose plus encore à l'époque moderne, dès la Renaissance, et semble toujours opératoire aujourd'hui. Dans des pages désormais célèbres consacrées à Velasquez, Michel Foucault a donné le modèle de l'analyse du lien structurel entre production d'illusions et spécularité (19-31). L'illusion tire son effet de réel de ce qu'elle naît dans une image qui se pense elle-même. Aussi, Velasquez parvient-il à matérialiser dans le hors-champ de la toile sa propre présence, à l'aide du jeu des regards et de la composition. De fait, la toile semble déborder sur la réalité, dans cette contiguïté entre le monde et sa représentation qui définit pour Barthes l'effet de réel (84-89). D’après Foucault, la production de l'illusion dans Les Ménines (Vélasquez, 1656) a un effet majeur : l'assignation du regard du spectateur.

$\mathrm{Au}$ moment où ils placent le spectateur dans le champ de leur regard, les yeux du peintre le saisissent, le contraignent à entrer dans le tableau, lui assignent un lieu à la fois privilégié et obligatoire, prélèvent sur lui sa lumineuse et visible espèce, et la projettent sur la surface inaccessible de la toile retournée. (Foucault 21)

Pareil mécanisme d'assignation immobilise le spectateur dans un ordre symbolique et concret - ici, les deux s'échangent dans la peinture - lui-même immuable. Mais ce dispositif est aussi celui qui dévoile au spectateur l'illusion. En témoigne l'analyse de Foucault ; pour le philosophe, cette assignation du regard demeure problématique dans la mesure où elle impose au spectateur une vérité à laquelle il peut difficilement 
échapper, quand bien même il en a conscience. D'après Colin MacCabe, le discours filmique impose en outre son ordre propre et sa signification au réel en se servant du spectateur et de sa position comme d'une fonction d'ordonnancement.

Grâce aux informations que nous glanons dans le récit, nous distinguons les discours des différents personnages et leur situation, et comparons ce qui s'y dit et ce que la narration nous révèle. La caméra nous montre ce qui arrive - elle dit la vérité en regard de laquelle nous évaluons les discours ${ }^{16}$.

La transparence du réalisme est due à la fonction qu'occupe le spectateur. La caméra reconstruit le réel et fournit une image non contradictoire de la réalité mais en dernière instance, c'est le spectateur qui valide la vérité particulière que lui fournit le film. Le circuit complexe par lequel s'élabore le réalisme fait que la manière dont le film impose son ordre et sa signification au réel n'est pas immédiatement perçue par le spectateur. Le réalisme, cette reconstruction du réel, est donc transparent, et avec lui l'idéologie. Colin MacCabe précise ce processus et la manière dont le texte filmique impose au réel son ordre et sa signification tout en contrôlant et en hiérarchisant les différents discours: "Le réalisme classique implique l'homogénéisation des discours par leur subordination à un discours dominant - lequel est assuré de sa domination par la sécurité et la transparence de son image ${ }^{17}$. " Le réalisme hollywoodien, selon MacCabe, consiste donc bien à refouler la dimension contradictoire du réel, à l'articuler harmonieusement de manière à lui ôter ses contradictions. De telles analyses concordent parfaitement avec les réflexions de Žižek (1997 7, 16-18) sur l'image fantasmatique. Par extrapolation, il devient possible d'affirmer que l'illusion créée par les effets spéciaux permet elle aussi de refouler la dimension contradictoire du réel, comme j'ai tâché de le montrer en analysant l'épilogue d'XXx: Return of Xander Cage. Bien sûr, il ne s'agit pas pour moi d'affirmer que toute représentation fondée sur la perspective a nécessairement pour effet de paralyser la réception critique du spectateur, mais plutôt de montrer à quelles conditions et selon quels mécanismes de telles représentations peuvent servir, dans certains cas, à diminuer la résistance critique du spectateur.

\section{Conclusion : comment briser les mécanismes idéologiques de l'illusion?}

Le caractère idéologique des effets spéciaux est déterminé par une série de paradoxes que j'ai tâché de faire apparaître ici. Cristallisés par l'exigence de photoréalisme, les mécanismes de la production idéologique en jeu dans les effets spéciaux forment un système, qui vise tout d'abord à dissimuler leur nature idéologique puis à oblitérer les antagonismes du réel et à assigner le spectateur à une position de regard précise dans un ordre symbolique, concret et politique donné. Les images de synthèse, procédés techniques et concrets, induisent un principe qui, lui, est abstrait: la spécularité cinématographique. Elle guide la création de l'illusion et la production idéologique. En se pensant elle-même, l'image produit un discours silencieux grâce auquel elle révèle son mode de production, tout en dissimulant ses propres fondements et mécanismes idéologiques. $\mathrm{Au}$ nombre de ces derniers, on compte l'illusion photoréaliste, l'assignation du regard du spectateur à un ordre et l'omniprésence d'un pouvoir absent mais partout agissant (Foucault 31). Mais si la spécularité est bien souvent utilisée comme une manière d'aveugler le spectateur, pris dans les rets des faux-semblants et 
les séductions de structures disposées en miroir, elle peut aussi être renversée en un instrument de lucidité lorsque les films décident de l'utiliser comme une forme critique.

Dans Transformers: Revenge of the Fallen, pourtant fort loin d'être un film subversif, les images de la séquence inaugurale sont montrées une deuxième fois sur les écrans d'étudiants tenant un site web d'informations alternatives. Ils mettent en doute la véracité de la version officielle - une fuite de gaz - en s'appuyant sur le caractère testimonial des images à l'écran. Les personnages s'appuient donc sur la rhétorique des thèses complotistes apparues dans le sillage du 11 septembre. Sam, le héros du film, seul personnage de la scène à pouvoir attester la réalité des images à l'écran, la dément cependant en invoquant la fiabilité de son regard. Voici le dialogue qui l'oppose aux trois étudiants acquis aux thèses complotistes :

- Looks fake to me.

- Dude, it's not fake. The internet is pure truth! Video doesn't lie!

- It looks fake...

- No man, I've seen them!

- Look, look, it's fake! Anybody could do it on any computer, okay? And I wasn't

there, so I can't comment or speculate ${ }^{18}$.

Puisque je n'ai pas été témoin des faits, dit-il en substance, je ne peux pas me prononcer sur la vérité de ces images. Or, le spectateur sait ici quelle version croire et valide la version des étudiants contre celle du héros qui cherche à dissimuler la vérité. Le discours sceptique est donc invalidé doublement, d'une part parce que l'on sait que les images sont fiables, d'autre part parce que l'on connaît les raisons pour lesquelles Sam les conteste. Parce qu'il valide l'illusion des effets spéciaux contre le mensonge du discours d'État, le récit nous invite, par une injonction contradictoire, à adopter un regard critique et à croire aux images de synthèse. En opposant une illusion fictionnelle (le discours d'État, dont le film nous dit qu'il est un mensonge) à une illusion véritable (les images de synthèse), le film nous fait adhérer à la production de faux-semblants, tout en feignant d'interroger les images sur un mode critique. Le conflit des rhétoriques sceptiques et complotistes au profit de ces dernières est ici l'alibi de la production idéologique.

La mise en abyme offre l'occasion d'une disputatio quant à la véracité des images. Celleci est figurée à l'écran mais c'est tout aussi bien le spectateur qui la mène avec luimême et qui se montre alternativement pyrrhonien (c'est-à-dire radicalement sceptique) et empiriste devant les images qui lui sont proposées. Or, comme l'a montré Aurélie Ledoux (174-206), dès lors que le spectateur adopte l'un ou l'autre de ces deux rapports à l'image, il devient un sujet politique indiscipliné, rejetant ce qu'on lui montre au nom de la vérité et de la raison ou croyant à ce qu'il voit pour nourrir son désir de révolte, comme le font les trois étudiants ou comme le fait la foule des émeutiers à la fin du Rollerball de McTiernan (MGM, 2002). La spécularité se pare ici des vertus d'un contre-pouvoir parce qu'elle ouvre, par sa complexité et ses replis, un espace de liberté herméneutique pour le spectateur, grâce auquel il échappe au discours de vérité que les images lui proposent. Quand elle n'est pas employée à renforcer l'illusion mimétique, la réflexivité des images peut donc servir au contraire à la renverser. La contradiction n'est ici qu'apparente, si l'on considère la spécularité moins comme un nécessaire facteur d'illusion (ce qu'elle peut être, mais pas toujours) que comme le lieu où se décide la tendance du récit à favoriser ou à freiner les fauxsemblants de l'image filmique. 
43 montage des images pour arracher le spectateur à l'illusion mimétique qui gouverne le récit et les effets spéciaux, selon le modèle brechtien de la distanciation (Brecht 57). Robert Stam a montré par ailleurs que le récit hollywoodien pouvait intégrer la distanciation brechtienne sans perdre pour autant son efficacité narrative. Ainsi des scènes de montage télévisuel dans The Insider (Révélations, Michael Mann, Touchstone, 1999) ou Rollerball, ou de la mise en doute de la mémoire à travers les écrans de Total Recall ou de Strange Days. Dans de tels moments, les films quittent la sphère des images de synthèse sophistiquées et policées pour renouer avec une pratique bricoleuse et frondeuse de l'assemblage d'images. Ils semblent alors dire que s'affranchir des illusions n'est possible qu'à la condition de s'émanciper des appareillages technologiques totalisants et hégémoniques, et invitent le spectateur à élire son propre mode de réception et de production d'images. Sans se retourner frontalement contre le système de production auquel ils appartiennent, ces films tentent en quelque sorte de créer des chemins de traverse ou des bifurcations au sein de l'appareil de production. Ils y ouvrent ainsi des images différentes, qui déterminent des modes de vision non spectaculaires. En refusant ainsi la norme technique hollywoodienne, ils contreviennent en sourdine à sa norme idéologique et se rêvent en grands films politiques consacrés aux images et à leurs manières de résister.

\section{BIBLIOGRAPHIE}

ALTHUSSER, Louis. « Idéologie et Appareils Idéologiques d'État (Notes pour une recherche) ». 1970. Positions (1964-1975). Paris : Éditions Sociales, 1976, p. 67-125.

BALIDES, Constance. «Immersion in the Virtual Ornament: Contemporary "Movie Ride" Films ». Rethinking Media Change: The Aesthetics of Transition. Dir. David Thorburn et Henry Jenkins. Cambridge, MA : MIT Press, 2003, p. 315-336.

BALTRUŠAITIS, Jurgis. Aberrations. Essai sur la légende des formes. 1957. Paris : Flammarion, 1983. ---. Anamorphoses ou « Thaumaturgus opticus ». 1955. Paris : Flammarion, 1984.

BARTHES, Roland. « L'effet de réel ». Communications, $n^{\circ}$ 11, 1968, p. 84-89.

DOI : 10.3406/comm.1968.1158

BAUDRILLARD, Jean. Simulacres et simulation. Paris : Galilée, 1981.

BELLER, Jonathan. The Cinematic Mode of Production: Attention Economy and the Society of the Spectacle. Lebanon, NH : Dartmouth College Press / University Press of New England, 2006.

BELLOÏ, Livio. Le regard retourné. Aspects du cinéma des premiers temps. Québec / Paris : Nota Bene / Méridiens Klincksieck, 2001.

BERTHOMIEU, Pierre. Le cinéma hollywoodien. Le temps du renouveau. Paris : Nathan, 2003.

BERTIN-MAGHIT, Jean-Pierre, dir. Une histoire mondiale des cinémas de propagande. Paris : Nouveau Monde, 2008. 
BESSY, Maurice. Les truquages au cinéma. Paris : Prisma, 1951.

BIDAUD, Anne-Marie. Hollywood et le rêve américain. Cinéma et idéologie aux États-Unis. 1994. Deuxième édition revue et corrigée. Paris : Armand Colin, 2012.

BOILLAT, Alain. Cinéma, machine à mondes. Essai sur les films à univers multiples. Chêne-Bourg : Georg, 2014.

BOLTER, Jay David et Richard GRUSIN. Remediation: Understanding New Media. Cambridge, MA : MIT Press, 2000.

BORDWELL, David, Janet STAIGER et Kristin THOMPSON. The Classical Hollywood Cinema: Film Style and Mode of Production to 1960. New York, NY : Columbia University Press, 1985. DOI :

$10.2307 / 1772109$

BRECHT, Bertolt. Petit organon pour le théâtre. 1948. Traduit de l'allemand par Jean Tailleur. Paris : L'Arche, 1990.

BUCI-GLUCKSMANN, Christine. La folie du voir. De l'esthétique baroque. Paris : Galilée, 1986.

BUKATMAN, Scott. « Spectacles, Attractions and Visual Pleasure ». The Cinema of Attractions Reloaded. Dir. Wanda Strauven. Amsterdam : Amsterdam University Press, 2006, p. 71-82.

CHKLOVSKI, Victor. L'art comme procédé. 1917. Traduit du russe par Régis Gayraud. Paris : Allia, 2008.

CLARK, Frank P. Special Effects in Motion Pictures: Some Methods for Producing Mechanical Special Effects. New York, NY : Society of Motion Picture and Television Engineers, 1966.

COTTA VAZ, Mark. Industrial Light and Magic: Into the Digital Realm. New York, NY : Random House, 1996.

DELEUZE, Gilles. « Le cerveau, c'est l'écran ». Cahiers du cinéma, nº 380, février 1986, p. 25-32.

DUBRAY, Joseph. « Large Aperture Lenses in Cinematography ». Transactions of the Society of Motion Picture Engineers, vol. 12, nº 33, 1928, p. 205-215.

DUCHAMP, Marcel. Notes. Ed. Pontus Hulten et Paul Matisse. Paris : Flammarion, 2008.

FOUCAULT, Michel. Les mots et les choses. Une archéologie des sciences humaines. Paris : Gallimard, 1966.

GARCIA, Thibaut. Qu'est-ce que le « virtuel » au cinéma ? Paris : L'Harmattan, 2009.

HAMUS-VALLÉE, Réjane. Les effets spéciaux. Paris : Éditions des Cahiers du cinéma, 2004.

---. « Préambule : qu'est-ce qu'un trucage ? Vers une définition impossible ». CinémAction, $\mathrm{n}^{\circ} 102$, 2002, p. 8-11.

HÉMARDINQUER, Pierre. Technique des effets spéciaux pour le film et la vidéo. Paris : Dujarric, 1980.

JAMESON, Fredric. L'inconscient politique. Le récit comme acte socialement symbolique. 1981. Traduit de l'anglais par Nicolas Vieillescazes. Paris : Questions théoriques, 2012.

KOPPES, Clayton R. et Gregory D. BLACK. « What to Show the World: The Office of War Information and Hollywood, 1942-1945 ». The Studio System. Dir. Janet Staiger. New Brunswick, NJ : Rutgers University Press, 1995, p. 279-297. DOI : 10.2307/1888275

KUSNIERZ, Mathias. « Faux-semblants et mises en abyme du blockbuster. Réflexions sur les effets idéologiques de la spécularité dans le cinéma américain contemporain ». Comprendre la mise en abyme. Arts et médias au second degré. Dir. Tonia Raus et Gian Maria Tore. Rennes : Presses universitaires de Rennes, 2019, p. 187-199. 
---. « L'usure iconique. Circulation et valeur des images dans le cinéma américain contemporain ». Transatlantica, $\mathrm{n}^{\circ} 2$ | 2016, 2017, journals.openedition.org/transatlantica/8330. Page consultée le 20 juin 2019.

---. « The Iconomics of Reflexivity: The Real Value of Images of Fiction in Contemporary American Cinema ». Narratologia, $\mathrm{n}^{\circ}$ 69. À paraître en 2020.

LASTRA, James. « Standards and Practices: Aesthetic Norms and Technological Innovation in the American Cinema ». The Studio System. Dir. Janet Staiger. New Brunswick, NJ : Rutgers University Press, 1995, p. 200-225

LÉCOLE SOLNYCHKINE, Sophie. « Cinéma et Légologie. L'effet-multivers du cube dans Pixels et Super 8 ». La Furia Umana, n 30, 2017, lafuriaumana.it/index.php/63-lfu-30/629-sophie-lecolesolnychkine-cinema-et-legologie-l-effet-multivers-du-cube-dans-pixels-et-super-8. Page consultée le 20 juin 2019.

LEDOUX, Aurélie. L'ombre d'un doute. Le cinéma américain contemporain et ses trompe-l'œil. Rennes : Presses universitaires de Rennes, 2012.

LEMIEUX, Philippe. L'image numérique au cinéma. Historique, esthétique et techniques d'une révolution technologique au cinéma. Paris : L'Harmattan, 2012.

LUNENFELD, Peter, dir. The Digital Dialectic: New Essays on New Media. Cambridge, MA : MIT Press, 1999.

MACCABE, Colin. « Realism and the Cinema: Notes on Some Brechtian Theses ». Screen, vol. 15, $\mathrm{n}^{\circ}$ 2, 1974, p. 7-27. DOI : 10.1093/screen/15.2.7

---. « Theory and Film: Principles of Realism and Pleasure ». Screen, vol. 17, n 3, 1976, p. 7-28.

DOI : $10.1093 /$ screen/17.3.7

MANOVICH, Lev. « What Is Digital Cinema? » The Digital Dialectic: New Essays on New Media. Dir. Peter Lunenfeld. Cambridge, MA : MIT Press, 1999, p. 172-192.

MCLUHAN, Marshall. Understanding Media: The Extensions of Man. New York, NY : McGraw-Hill, 1964.

MEISEL, Louis K. Photorealism. New York, NY : Abradale / Abrams, 1989.

MERLEAU-PONTY, Maurice. Phénoménologie de la perception. Paris : Gallimard, 1945.

DOI : $10.14375 /$ NP.9782070293377

MOMCILOVIC, Jérôme. Prodiges d'Arnold Schwarzenegger. Nantes : Capricci, 2016.

MONTE, James K. 22 Realists. New York, NY : Whitney Museum of American Art, 1970.

MULVEY, Laura. « Visual Pleasure and Narrative Cinema ». Screen, vol. 16, n 3, 1975, p. 6-18. DOI : $10.1093 /$ screen/16.3.6

NACACHE, Jacqueline. «War Comes to America : le cinéma hollywoodien entre effort de guerre et propagande (1939-1945) ». Une histoire mondiale des cinémas de propagande. Dir. Jean-Pierre BertinMaghit. Paris : Nouveau Monde, 2008, p. 363-402.

NDALIANIS, Angela. Neo-Baroque Aesthetics and Contemporary Entertainment. Cambridge, MA : MIT Press, 2004. DOI : 10.7551/mitpress/4912.001.0001

ODELLO, Laura, dir. Blockbuster. Philosophie et cinéma. Paris : Les Prairies ordinaires, 2013.

PACI, Viva. « Cinéma de synthèse et cinéma des premiers temps. Des correspondances examinées à la loupe du système des attractions ». Cinéma\&Cie, $\mathrm{n}^{\circ}$ 5, 2004, p. 112-114. 
---. « La persistance des attractions ». Cinéma\&Cie, $\mathrm{n}^{\circ}$ 3, 2003, p. 56-63.

---. « The Attraction of the Intelligent Eye: Obsessions with the Vision Machine in Early Film Theories ». The Cinema of Attractions Reloaded. Dir. Wanda Strauven. Amsterdam : Amsterdam University Press, 2006, p. 121-137.

PANOFSKY, Erwin. « Les antécédents idéologiques de la calandre Rolls-Royce ». 1963. Trois essais sur le style. Traduit de l'anglais par Bernard Turle. Paris : Gallimard, 1996, p. 147-189.

PRIGENT, Christian. « Aux grands anamorphoseurs ». L'ennemi, nº 1, 1982, p. 132-143.

PRINCE, Stephen. « True Lies: Perceptual Realism, Digital Images, and Film Theory ». Film Quarterly, vol. 49, nº 3, 1996, p. 27-37. DOI : 10.2307/1213468

RANCIÈRE, Jacques. Le destin des images. Paris : La Fabrique, 2003.

RODOWICK, David Norman. The Virtual Life of Film. Cambridge, MA : Harvard University Press, 2007. DOI : $10.4159 / 9780674042834$

SARTRE, Jean-Paul. Qu'est-ce que la littérature? 1948. Paris : Gallimard, 1993.

SCHATZ, Thomas. Old Hollywood / New Hollywood: Ritual, Art, and Industry. Ann Arbor, MI : UMI Research Press, 1976.

SOBCHACK, Vivian. The Adress of the Eye: A Phenomenology of Film Experience. Princeton, $\mathrm{NJ}$ : Princeton University Press, 1992.

STAIGER, Janet, dir. The Studio System. New Brunswick, NJ : Rutgers University Press, 1995.

STAM, Robert. Reflexivity in Film and Literature: From Don Quixote to Jean-Luc Godard. Ann Arbor, MI : UMI Research Press, 1985.

STRAUVEN, Wanda, dir. The Cinema of Attractions Reloaded. Amsterdam : Amsterdam University Press, 2006. DOI : 10.5117/9789053569450

THORBURN, David et Henry JENKINS, dir. Rethinking Media Change: The Aesthetics of Transition. Cambridge, MA : MIT Press, 2003.

TOMASOVIC, Dick. « The Hollywood Cobweb: New Laws of Attractions (The Spectacular Mechanics of Blockbusters) ». The Cinema of Attractions Reloaded. Dir. Wanda Strauven. Amsterdam : Amsterdam University Press, 2006, p. 309-320.

WILLIAMS, Christopher. «After the Classic, the Classical and Ideology: The Differences of Realism ». Screen, vol. 35, nº 3, 1994, p. 275-292. DOI : 10.1093/screen/35.3.275

WORLAND, Rick. « OWI Meets the Monsters: Hollywood Horror Films and War Propaganda, 1942 to 1945 ». Cinema Journal, vol. 37, nº 1, 1997, p. 47-65.

DOI : $10.2307 / 1225689$

ŽIŽEK, Slavoj. The Plague of Fantasies. Londres : Verso, 1997.

---. « Un exercice d'idéologie politiquement correct ». Cahiers du cinéma, n 654, mars 2010, p. 66-69.

\section{Filmographie}

20,000 Leagues Under the Sea (20.000 lieues sous les mers). Réal. Richard Fleischer. Scénario d'Earl Felton. Avec Kirk Douglas (Ned Land), James Mason (Capitaine Nemo), Paul Lukas (Professeur Pierre Aronnax), Peter Lorre (Conseil), Robert J. Wilke (Commandant en second du Nautilus). Dir. 
photo. Elmo Williams. Montage John Meehan. Musique de Paul J. Smith. États-Unis, Walt Disney Productions, 1954.

Apocalypse Now. Réal. Francis Ford Coppola. Scénario de John Milius, Francis Ford Coppola et Michael Herre. Avec Martin Sheen (Capitaine Benjamin L. Willard), Marlon Brando (Colonel Walter E. Kurtz), Robert Duval (Lieutenant-colonel Bill Kilgore), Frederic Forrest (Jay « Chef » Hicks, mécanicien et tireur de poupe du bateau), Albert Hall (Lieutenant George Philipps, commandant du bateau). Dir. photo. Vittorio Storaro. Montage Walter Murch, Gerald B. Greenberg et Lisa Fruchtmann; Michael Kirchberger (version redux). Musique de Carmine Coppola, Francis Ford Coppola, Mickey Hart et Randy Hansen. États-Unis, United Artists, 1979.

Avatar. Réal. James Cameron. Scénario de James Cameron. Avec Sam Worthington (Jake Sully), Sigourney Weaver (Docteur Grace Augustine), Steven Lang (Colonel Miles Quaritch), Michelle Rodríguez (Trudy Chacon). Dir. photo. Mauro Fiore. Montage James Cameron, John Refoua et Steven E. Rivkin. Musique de James Horner. États-Unis, Twentieth Century Fox, 2009.

Cleopatra (Cléopâtre). Réal. Joseph L. Mankiewicz. Scénario de Joseph L. Mankiewicz, Ranald MacDougall et Sidney Buchman. Avec Elizabeth Taylor (Cléopâtre), Richard Burton (Marc Antoine), Rex Harrison (Jules César), Roddy McDowall (Octave). Dir. photo. Leon Shamroy et Jack Hildyard. Montage Dorothy Spencer et Elmo Williams. Musique d'Alex North. États-Unis, Twentieth Century Fox, 1963.

Destination Moon (Destination... Lune !). Réal. Irving Pichel. Scénario d'Alford Van Ronkel, James O'Hanlon et Robert A. Heinlein. Avec John Archer (Jim Barnes), Walter Anderson (Docteur Charles Cargraves), Tom Powers (Général Thayer), Dick Wesson (Joe Sweeney), Erin O’Brien-Moore (Emily Cargraves). Dir. photo. Lionel Lindon. Montage Duke Goldstone. Musique de Leith Stevens. ÉtatsUnis, Eagle-Lion, 1950.

Détective Dee. Le Mystère de la flamme fantôme. Réal. Tsui Hark. Scénario de Chen Kuo-fu. Avec Andy Lau (Di Renjie, détective Dee), Carina Lau (Wu Zetian, impératrice de Chine), Tony Leung Ka-fai (Shatuo Zhong). Dir. photo. Parkie Chan et Chan Chi-ying. Montage Yau Chi-wai. Musique de Peter Kam. Hong-Kong, Huayi Brothers Media, 2010.

Détective Dee. La Légende du dragon des mers. Réal. Tsui Hark. Scénario de Chiang Chia-lu et Chen Kuo-fu. Avec Mark Chao (Di Renjie, détective Dee), Carina Lau (Wu Zetian, impératrice de Chine), Chen Kun (Wang Pu). Dir. photo. Choi Sung-fai. Montage Yu Bo-yang. Musique de Kenji Kawai. Hong-Kong, Huayi Brothers Media, 2013.

Détective Dee. La Légende des rois célestes. Réal. Tsui Hark. Scénario de Chiang Chia-lu. Avec Mark Chao (Di Renjie, détective Dee), Carina Lau (Wu Zetian, impératrice de Chine), Chien Sheng (Tang Gaozong, empereur de Chine). Dir. photo. Choi Sung-fai. Montage Tsui Hark et Lin Li. Musique de Kenji Kawai. Hong-Kong, Huayi Brothers Media, 2018.

Edge of Tomorrow. Réal. Doug Liman. Scénario de Christopher McQuarrie, Jez Butterworth et JohnHenry Butterworth. Avec Tom Cruise (Commandant William « Bill » Cage), Emily Blunt (Sergent Rita Rose Vrataski), Brendan Gleeson (Général Brigham), Bill Paxton (Sergent maître Bartolome Farell). Dir. photo. Dion Beebe. Montage James Herbert. Musique de Christophe Beck. États-Unis, Warner Bros., 2014.

Geostorm. Réal. Dean Devlin. Scénario de Dean Devlin et Paul Guyot. Avec Gerard Butler (Jake Lawson), Jim Sturgess (Max Lawson), Abbie Cornish (Sarah Wilson). Dir. photo. Roberto Schaefer. Montage Ron Rosen. Musique de Lorne Balfe. États-Unis, Warner Bros., 2017.

Ghost in the Shell. Réal. Mamoru Oshii. Scénario de Kazunori Itô. Avec les voix d'Atsuko Tanaka (Major Motoko Kusanagi), Akio Ōtsuka (Batou), Tamio Ōki (Aramaki), Iemasa Tayumi (Projet 2501, 
alias Puppet Master), Kōichi Yamadera (Togusa), Tesshō Genda (Nakamura). Dir. photo. Hisao Shirai. Montage Shûichi Kakesu. Musique de Kenji Kawai. Japon, Kodashan, 1995.

Ghost in the Shell. Réal. Rupert Sanders. Scénario de Jamie Moss, William Wheeler et Ehren Kruger. Avec Scarlett Johansson (Major Motoko Kusanagi), Pilou Asbæk (Batou), 'Beat' Takeshi Kitano (Aramaki), Michael Pitt (Kuze), Juliette Binoche (Docteur Ouelet), Chin Han (Togusa), Yutaka Izumihara (Saito). Dir. photo. Jess Hall. Montage Billy Rich et Neil Smith. Musique de Lorne Balfe et Clint Mansell. États-Unis, DreamWorks, 2017.

Inception. Réal. Christopher Nolan. Scénario de Christopher Nolan. Avec Leonardo DiCaprio (Dominic « Dom » Cobb), Marion Cotillard (Mallorie « Mal » Cobb), Joseph Gordon-Levitt (Arthur), Ellen Page (Ariane), Ken Watanabe (Saito), Tom Hardy (Eames), Cillian Murphy (Robert Michael Fischer, Jr.), Michael Caine (Professeur Miles). Dir. photo. Wally Pfister. Montage Lee Smith. Musique de Hans Zimmer. États-Unis, Warner Bros., 2010.

Jaws (Les Dents de la mer). Réal. Steven Spielberg. Scénario de Peter Benchley et Carl Gottlieb. Avec Roy Scheider (Brody), Robert Shaw (Quint), Richard Dreyfuss (Hooper). Dir. photo. Bill Butler. Montage Verna Fields. Musique de John Williams. États-Unis, Universal, 1975.

Jurassic Park. Réal. Steven Spielberg. Scénario de Michael Crichton et David Koepp. Avec Jeff Goldblum (Malcolm), Laura Dern (Ellie), Sam Neill (Grant), Richard Attenborough (Hammond). Dir. photo. Dean Cundey. Montage Michael Kahn. Musique de John Williams. États-Unis, Universal, 1993.

Letters from Iwo Jima (Lettres d'Iwo Jima). Réal. Clint Eastwood. Scénario d'Iris Yamashita et Paul Haggis. Avec Ken Watanabe (Général Kuribayashi), Kazunari Ninomiya (Saigo), Tsuyoshi Ihara (Baron Nishi), Ryo Kase (Shimizu). Dir. photo. Tom Stern. Montage Joel Cox et Gary Roach. Musique de Kyle Eastwood et Michael Stevens. États-Unis, Warner Bros., 2006.

Minority Report. Réal. Steven Spielberg. Scénario de Scott Frank et Jon Cohen. Avec Tom Cruise (John Anderton), Colin Farrell (Danny Witwer), Max von Sydow (Lamar Burgess), Samantha Morton (Agatha). Dir. photo. Janusz Kaminski. Montage Michael Kahn. Musique de John Williams. États-Unis, Twentieth Century Fox, 2002.

Mission: Impossible - Fallout. Réal. Christopher McQuarrie. Scénario de Christopher McQuarrie. Avec Tom Cruise (Ethan Hunt), Simon Pegg (Benji Dunn), Henry Cavill (August Walker), Ving Rhames (Luther Stickell), Rebecca Ferguson (Ilsa Faust), Sean Harris (Solomon Lane). Dir. photo. Rob Hardy. Montage Eddie Hamilton. Musique de Lorne Balfe. États-Unis, Paramount, 2018.

Rogue One: A Star Wars Story. Réal. Gareth Edwards. Scénario de Chris Weitz, Tony Gilroy, John Knoll et Gary Whitta. Avec Felicity Jones (Jyn Erso), Diego Luna (Cassian Andor), Alan Tudik (K-2SO). Dir. photo. Greig Fraser. Montage John Gilroy, Colin Goudie et Jabez Olssen. Musique de John Williams et Michael Giacchino. États-Unis, Lucasfilm, 2016.

Rollerball. Réal. John McTiernan. Scénario de Larry Ferguson et John Pogue. Avec Chris Klein (Jonathan Cross), LL Cool J (Marcus Ridley), Jean Reno (Alexis Petrovich), Rebecca Romijn-Stamos (Aurora). Dir. photo. Steve Mason. Montage Robert K. Lambert et John Wright. Musique d'Éric Serra. États-Unis, MGM, 2002.

Solo: A Star Wars Story. Réal. Ron Howard. Scénario de Jonathan Kasdan et Lawrence Kasdan. Avec Alden Ehrenreich (Han Solo), Joonas Suotamo (Chewbacca), Woody Harrelson (Beckett). Dir. photo. Bradford Young. Montage Pietro Scalia. Musique de John Powell. États-Unis, Lucasfilm, 2018.

Spider-Man. Réal. Sam Raimi. Scénario de David Koepp. Avec Tobey Maguire (Peter Parker / Spider-Man), Kirsten Dunst (Mary Jane Watson), Willem Defoe (Norman Osborn / Green 
Goblin), James Franco (Harry Osborn), Cliff Robertson (Ben Parker). Dir. photo. Don Burgess. Montage Arthur Coburn et Bob Murawski. Musique de Danny Elfman. États-Unis, Columbia, 2002. Spider-Man 2. Réal. Sam Raimi. Scénario d'Alfred Gough, Miles Millar, Michael Chabon et Alvin Sargent. Avec Tobey Maguire (Peter Parker / Spider-Man), Kirsten Dunst (Mary Jane Watson), Alfred Molina (Docteur Otto Octavius / Doctor Octopus), James Franco (Harry Osborn), Rosemary Harris (May Parker). Dir. photo. Bill Pope. Montage Bob Murawski. Musique de Danny Elfman. États-Unis, Columbia, 2004.

Spider-Man 3. Réal. Sam Raimi. Scénario de Sam Raimi, Ivan Raimi et Alvin Sargent. Avec Tobey Maguire (Peter Parker / Spider-Man), Kirsten Dunst (Mary Jane Watson), James Franco (Harry Osborn / New Goblin), Thomas Haden Church (Flint Marko / Sandman), Topher Grace (Eddie Brock / Venom), Bryce Dallas Howard (Gwen Stacy), Rosemary Harris (May Parker). Dir. photo. Bill Pope. Montage Bob Murawski. Musique de Christopher Young. États-Unis, Columbia, 2007.

Star Wars: Episode IV - A New Hope (Star Wars : Épisode IV - Un nouvel espoir, paru en France en 1977 sous le titre La Guerre des étoiles). Réal. George Lucas. Scénario de George Lucas. Avec Harrison Ford (Han Solo), Mark Hamill (Luke Skywalker), Carrie Fisher (Princesse Leia), Alec Guiness (ObiWan Kenobi), David Prowse (Darth Vader). Dir. photo. Gilbert Taylor. Montage Richard Chew, T. M. Christopher, Paul Hirsch, Marcia Lucas et George Lucas. Musique de John Williams. États-Unis, Lucasfilm, 1977.

Star Wars: Episode V - The Empire Strikes Back (Star Wars : Épisode V - L'Empire contre-attaque, paru en France en 1980 sous le titre L'Empire contre-attaque). Réal. Irving Kershner. Scénario de Leigh Brackett, Lawrence Kasdan et George Lucas. Avec Harrison Ford (Han Solo), Mark Hamill (Luke Skywalker), Carrie Fisher (Princesse Leia), Alec Guiness (Obi-Wan Kenobi), David Prowse (Darth Vader). Dir. photo. Peter Suschitzky. Montage T. M. Christopher, Paul Hirsch, Marcia Lucas et George Lucas. Musique de John Williams. États-Unis, Lucasfilm, 1980.

Star Wars: Episode VI - Return of the Jedi (Star Wars : Épisode VI - Le Retour du Jedi, paru en France en 1983 sous le titre Le Retour du Jedi). Réal. Richard Marquand. Scénario de Lawrence Kasdan et George Lucas. Avec Harrison Ford (Han Solo), Mark Hamill (Luke Skywalker), Carrie Fisher (Princesse Leia), Alec Guiness (Obi-Wan Kenobi), Sebastian Shaw (Anakin Skywalker), David Prowse (Darth Vader). Dir. photo. Alan Hume et Alec Mills. Montage Sean Barton, T. M. Christopher, Duwayne Dunham, Marcia Lucas et George Lucas. Musique de John Williams. ÉtatsUnis, Lucasfilm, 1983.

Star Wars: Episode I - The Phantom Menace (Star Wars : Épisode I - La Menace fantôme). Réal. George Lucas. Scénario de George Lucas. Avec Ewan McGregor (Obi-Wan Kenobi), Liam Neeson (Qui-Gon Jinn), Nathalie Portman (Amidala / Padmé), Jake Lloyd (Anakin Skywalker), Ian McDiarmid (Sénateur Palpatine). Dir. photo. David Tattersall. Montage Ben Burtt et Paul Martin Smith. Musique de John Williams. États-Unis, Lucasfilm, 1999.

Star Wars: Episode II - Attack of the Clones (Star Wars : Épisode II - L'Attaque des clones). Réal. George Lucas. Scénario de Jonathan Hales et George Lucas. Avec Ewan McGregor (Obi-Wan Kenobi), Liam Neeson (Qui-Gon Jinn), Nathalie Portman (Padmé), Hayden Christensen (Anakin Skywalker), Samuel L. Jackson (Mace Windu), Ian McDiarmid (Chancelier suprême Palpatine / Darth Sidious), Christopher Lee (Comte Dooku / Darth Tyranus). Dir. photo. David Tattersall. Montage Ben Burtt et George Lucas. Musique de John Williams. États-Unis, Lucasfilm, 2002.

Star Wars: Episode III - Revenge of the Sith (Star Wars : Épisode III - La Revanche des Sith). Réal. George Lucas. Scénario de George Lucas. Avec Ewan McGregor (Obi-Wan Kenobi), Nathalie Portman (Padmé), Hayden Christensen (Anakin Skywalker), Samuel L. Jackson (Mace Windu), Ian McDiarmid (Chancelier suprême Palpatine / Darth Sidious), Christopher Lee (Comte 
Dooku / Darth Tyranus). Dir. photo. David Tattersall. Montage Roger Barton et Ben Burtt. Musique de John Williams. États-Unis, Lucasfilm, 2005.

Star Wars: Episode VII - The Force Awakens (Star Wars : Épisode VII - Le Réveil de la Force). Réal. J. J. Abrams. Scénario de Lawrence Kasdan, J. J. Abrams et Michael Arndt. Avec Daisy Ridley (Rey), Carrie Fisher (Princesse Leia), Harrison Ford (Han Solo), Adam Driver (Kylo Ren), Mark Hamill (Luke Skywalker), John Boyega (Finn), Oscar Isaac (Poe Dameron). Dir. photo. Dan Mindel. Montage Maryann Brandon et Mary Jo Markey. Musique de John Williams. États-Unis, Lucasfilm, 2015.

Star Wars: Episode VIII - The Last Jedi (Star Wars : Épisode VIII - Les Derniers Jedi). Réal. Rian Johnson. Scénario de Rian Johnson. Avec Daisy Ridley (Rey), Carrie Fisher (Princesse Leia), Adam Driver (Kylo Ren), Mark Hamill (Luke Skywalker), John Boyega (Finn), Oscar Isaac (Poe Dameron), Andy Serkis (Snoke). Dir. photo. Steve Yedlin. Montage Bob Ducsay. Musique de John Williams. ÉtatsUnis, Lucasfilm, 2017.

Star Wars: Episode IX - The Rise of Skywalker (Star Wars : Épisode IX - L'Ascension de Skywalker). Réal. J. J. Abrams. Scénario de J. J. Abrams, Chris Terrio, Colin Trevorrow et Derek Conolly. Avec Daisy Ridley (Rey), Carrie Fisher (Princesse Leia), Adam Driver (Kylo Ren), Mark Hamill (Luke Skywalker), John Boyega (Finn), Oscar Isaac (Poe Dameron), Billie Lourd (Lieutenant Connix). Dir. photo. Dan Mindel. Montage Maryann Brandon et Stefan Grube. Musique de John Williams. ÉtatsUnis, Lucasfilm, 2019.

Strange Days. Réal. Kathryn Bigelow. Scénario de James Cameron et Jay Cocks. Avec Ralph Fiennes (Lenny Nero), Angela Bassett (Lornette « Mace» Mason), Juliette Lewis (Faith Justin), Tom Sizemore (Max Peltier). Dir. photo. Matthew F. Leonetti. Montage Howard E. Smith et James Cameron. Musique de Graeme Revell. États-Unis, Lightstorm Entertainment, 1995.

Terminator 2: Judgement Day (Terminator 2 : Le Jugement dernier). Réal. James Cameron. Scénario de James Cameron et William Wisher. Avec Arnold Schwarzenegger (T-800), Linda Hamilton (Sarah Connor), Edward Furlong (John Connor), Robert Patrick (T-1000). Dir. photo. Adam Greenberg. Montage Conrad Buff IV, Dody Dorn, Mark Goldblatt et Richard A. Harris. Musique de Brad Fiedel. États-Unis, TriStar Pictures, 1991.

The Birds (Les Oiseaux). Réal. Alfred Hitchcock. Scénario d'Evan Hunter. Avec Tippi Hedren (Melanie Daniels), Rod Taylor (Mitch Brenner), Jessica Tandy (Lydia Brenner). Dir. photo. Robert Burks. Montage George Tomasini. Musique (sound design) de Bernard Hermann et Remi Gassmann. États-Unis, Universal, 1963.

The Bourne Supremacy (La Mort dans la peau). Réal. Paul Greengrass. Scénario de Tony Gilroy. Avec Matt Damon (Jason Bourne), Franka Potente (Marie), Brian Cox (Ward Abbott), Julia Stiles (Nicky), Joan Allen (Pamela Landy). Dir. photo. Oliver Wood. Montage Richard Pearson et Christopher Rouse. Musique de John Powell. États-Unis, Universal, 2004.

The Day After Tomorrow (Le Jour d'après). Réal. Roland Emmerich. Scénario de Roland Emmerich et Jeffrey Nachmanoff. Avec Jake Gyllenhaal (Sam Hall), Dennis Quaid (Jack Hall), Emmy Rossum (Laura Chapman). Dir. photo. Ueli Steiger. Montage David Brenner. Musique de Harald Kloser. États-Unis, Twentieth Century Fox, 2004.

The Devil at 4 O'Clock (Le Diable à 4 heures). Réal. Mervyn LeRoy. Scénario de Liam O’Brien. Avec Spencer Tracy (Matthew Doonan), Frank Sinatra (Harry), Kerwin Mathews (Joseph Perreau). Dir. photo. Joseph F. Biroc. Montage Charles Nelson. Musique de George Duning. États-Unis, Columbia, 1961.

The Insider (Révélations). Réal. Michael Mann. Scénario de Michael Mann et Eric Roth. Avec Al Pacino (Lowell Bergman), Russell Crowe (Jeffrey Wigand), Christopher Plummer (Mike Wallace), 
Diane Venora (Liane Wigand). Dir. photo. Dante Spinotti. Montage William Goldenberg, David Rosenbloom et Paul Rubell. Musique de Lisa Gerrard, Pieter Bourke, Michael Brook et Gustavo Santaolalla. États-Unis, Buena Vista Pictures, 1999.

The Lord of the Rings: The Fellowship of the Ring (Le Seigneur des anneaux. La Communauté de l'anneau). Réal. Peter Jackson. Scénario de Fran Walsh, Philippa Boyens et Peter Jackson. Avec Elijah Wood (Frodo), Ian McKellen (Gandalf), Orlando Bloom (Legolas), Viggo Mortensen (Aragorn). Dir. photo. Andrew Lesnie. Montage John Gilbert. Musique de Howard Shore. États-Unis et Nouvelle-Zélande, New Line Cinema, 2001.

The Lord of the Rings: The Two Towers (Le Seigneur des anneaux. Les Deux Tours). Réal. Peter Jackson. Scénario de Fran Walsh, Philippa Boyens, Steven Sinclair et Peter Jackson. Avec Elijah Wood (Frodo), Ian McKellen (Gandalf), Orlando Bloom (Legolas), Viggo Mortensen (Aragorn). Dir. photo. Andrew Lesnie. Montage Michael Horton. Musique de Howard Shore. États-Unis et NouvelleZélande, New Line Cinema, 2002.

The Lord of the Rings: The Return of the King (Le Seigneur des anneaux. Le Retour du roi). Réal. Peter Jackson. Scénario de Fran Walsh, Philippa Boyens et Peter Jackson. Avec Elijah Wood (Frodo), Ian McKellen (Gandalf), Orlando Bloom (Legolas), Viggo Mortensen (Aragorn). Dir. photo. Andrew Lesnie. Montage Jamie Selkirk. Musique de Howard Shore. États-Unis et Nouvelle-Zélande, New Line Cinema, 2003.

The Lost World (Le Monde perdu). Réal. Irwin Allen. Scénario de Charles Bennett et Irwin Allen. Avec Michael Rennie (Lord John Roxton), Jill St. John (Jennifer Holmes), David Edison (Ed Malone). Dir. photo. Winton C. Hoch. Montage Hugh S. Fowler. Musique de Paul Sawtell et Bert Shefter. ÉtatsUnis, Twentieth Century Fox, 1960.

The Ten Commandments (Les Dix Commandements). Réal. Cecil B. DeMille. Scénario de Jesse Lasky, Jr., Æneas MacKenzie, Jack Gariss et Fredric M. Frank. Avec Charlton Heston (Moses), Yul Brynner (Rameses), Anne Baxter (Nefretiri), Edward G. Robinson (Dathan). Dir. photo. Loyal Griggs, W. Wallace Kelley, J. Peverell Marley et John F. Warren. Montage Anne Bauchens. Musique d'Elmer Bernstein. États-Unis, Paramount, 1956.

Total Recall. Réal. Paul Verhoeven. Scénario de Ronald Shusett, David O’Bannon et Gary Goldman. Avec Arnold Schwarzenegger (Douglas Quaid), Sharon Stone (Lori Quaid), Michael Ironside (Richter), Rachel Ticotin (Melina). Dir. photo. Jost Vacano. Montage Carlos Puente et Frank J. Urioste. Musique de Jerry Goldsmith. États-Unis, TriStar Pictures, 1990.

Transformers: Revenge of the Fallen (Transformers 2 : La Revanche). Réal. Michael Bay. Scénario de Roberto Orci, Alex Kurtzman et Ehren Kruger. Avec Shia Labeouf (Sam Witwicky), Megan Fox (Mikaela Banes), Ramon Rodriguez (Leo Spitz). Dir. photo. Ben Seresin. Montage Roger Barton, Tom Muldoon, Joel Negron et Paul Rubell. Musique de Steve Jablonsky. États-Unis, Paramount, 2009.

True Lies. Réal. James Cameron. Scénario de James Cameron. Avec Arnold Schwarzenegger (Harry Tasker / Harry Rinquest), Jamie Lee Curtis (Helen Elizabeth Tasker), Tom Arnold (Albert Gibson), Bill Paxton (Simon Carlos), Art Malik (Salim Abu Aziz), Tia Carrere (Juno Skinner). Dir. photo. Russell Carpenter. Montage Conrad Buff IV, Mark Goldblatt et Richard A. Harris. Musique de Brad Fiedel. États-Unis, Lightstorm Entertainment, 1994.

Under the Skin. Réal. Jonathan Glazer. Scénario de Walter Campbell, Jonathan Glazer et Milo Addica. Avec Scarlett Johansson (la femme), Paul Brannigan (Andrew). Dir. photo. Daniel Landin. Montage Paul Watts. Musique de Mica Levi. Grande Bretagne, Film4, 2013.

War of the Worlds (La Guerre des mondes). Réal. Byron Haskin. Scénario de Barré Lyndon. Avec Gene Barry (Docteur Clayton Forrester), Ann Robinson (Sylvia Van Buren), Robert Cornthwaite 
(Docteur Pryor). Dir. photo. George Barnes. Montage Everett Douglas. Musique de Leith Stevens. États-Unis, Paramount, 1953.

When Worlds Collide (Le Choc des mondes). Réal. Rudolph Maté. Scénario de Sydney Boehm. Avec Richard Derr (David Randall), Barbara Rush (Joyce Hendron), Peter Hansen (Docteur Tony Drake). Dir. photo. W. Howard Greene et John F. Seitz. Montage Arthur P. Schmidt. Musique de Leith Stevens. États-Unis, Paramount, 1951.

xXx: Return of Xander Cage ( $x$ Xx: Reactivated). Réal. D. J. Caruso. Scénario de Rich Wilkes et F. Scott Frazier. Avec Vin Diesel (Xander Cage), Donnie Yen (Xiang), Deepika Padukone (Serena Unger), Samuel L. Jackson (Augustus Gibbons), Nina Dobrev (Becky Clearidge), Ice Cube (Darius Stone), Toni Collette (Jane Marke). Dir. photo. Russell Carpenter. Montage Vince Filippone et Jim Page. Musique de Robert Lydecker et Brian Tyler. États-Unis, Paramount, 2017.

\section{Fuvres d'art}

REMBRANDT (Rembrandt Harmenszoon van Rijn, dit Rembrandt). La Leçon d'anatomie du docteur Tulp (De anatomische les van dr. Nicolaes Tulp). 1632. Huile sur toile, 169,5 x 216,5 cm. Mauritshuis, La Haye.

VÉLASQUEZ, Diego. Les Ménines (Las Meninas). 1656. Huile sur toile, 318 x 276 cm. Musée du Prado, Madrid.

\section{NOTES}

1. Destination Moon et When Worlds Collide sont évoqués à la page 110, War of the Worlds à la page 145, 20,000 Leagues Under the Sea à la page 89, The Lost World à la page 23, The Devil at 4 O'Clock aux pages 139, 153 et 214 et The Birds à la page 92 (Clark, 1966).

2. "This period saw the birth of "invisible" or "psychological" editing which was the basis of Hollywood's classic narrative style, and which generated a degree of audience identification and engagement far surpassing that in the more technically primitive one-reelers. » (Schatz 34). Ici comme ailleurs, sauf mention contraire, c'est moi qui traduis.

3. "If the address of the spectator uses little the look at camera by actors, the narrative break and the reminder of the spectator's status is revealed by hyperbolic camera movements, but also, among other things, by a series of referential shots (the surgical scene of Octopus's tentacles amputation, a wink at Raimi's faithful spectators in the direction of his previous films), and the recourse to the burlesque close-up (the insert of the spider which bites Peter into SM1, a real visual moment, autonomous, striking and comic). » [sic]

4. Bien que cette expression soit difficilement traduisible, on pourrait parler, en français, de « séances pour s'évader ».

5. S'il m'est permis de livrer ici une anecdote personnelle, j'ai été frappé, au moment de revoir le film pour écrire cet article, de constater combien j'avais été aveugle à toute cette dimension lorsque j'ai vu True Lies pour la première fois à l'âge de 13 ou 14 ans. C'est peut-être à partir d'une telle cécité que travaille la fonction idéologique des films américains.

6. «But, as this essay has suggested, some of these representations, while being referentially unreal, are perceptually realistic $»$ (Prince 35).

7. «It is now possible to cut, blend, stretch and stitch digitized film images into something that has perfect photographic credibility, although it was never actually filmed (Manovich 175).

8. Dans les scènes d'action de Ghost in the Shell, Scarlett Johansson est filmée en motion capture et son camouflage est appliqué en post-production comme une peau numérique. 
9. "Good special effects create the illusion of reality without the audience becoming immediately aware of the deception.» (Clark 27)

10. L'article de Joseph Dubray atteste la précocité du discours sur la technologie à Hollywood.

11. "Page after page in scientific and industry journals emphatically promote various aesthetic models, but why? What function did the articulation of aesthetic standards and norms play? Far from being incidental, technicians almost seem nearly obsessed with discussing their positions on questions of representational illusion, accuracy, propriety, and validity, and, almost uniformly, the standards they assert are justified in terms of a transparent realism. » (Lastra 203) 12. Certes, la prouesse technique de Jurassic Park a plutôt consisté à mêler harmonieusement images numériques et animatroniques (des robots manipulés à distance par des câbles).

13. "Our times are marked by radical transitions similar to those in the seventeenth century: Advances in optical technology have both aided in making the previously invisible visible and constructed alternate realities that build digital environments that offer sensorially authentic experiences. » (Ndalianis 179)

14. «C'est pas compliqué : lattez les méchants, chopez la fille et au passage, tâchez d'avoir l'air cool.»

15. Bien sûr, le gain de photoréalisme de ces images est en quelque sorte compensé par la connaissance croissante que le public a de ces techniques et par le fait qu'il les discerne de mieux en mieux - ce qui ne l'empêche pourtant pas de tenir pour « réaliste » ce qu'il voit, et d'y croire.

16. "Through the knowledge we gain from the narrative we can split the discourses of the various characters from their situation and compare what is said in these discourses with what has been revealed to us through narration. The camera shows us what happens - it tells the truth against which we can measure the discourses. » (MacCabe, 1974 10)

17. "Classical realism [...] involves the homogeneisation of different discourses by their relation to one dominant discourse - assured of its domination by the security and transparency of its image. The fact that one such practice involves this homogeneisation is a matter of ideological and political but not normative interest. » (MacCabe, 1976 12)

18. « - Ça a l'air faux. - C'est pas faux mon pote. Internet, c'est la pure vérité. La vidéo ne ment pas. - Mais ça a l'air faux... - Mais non mec, je les ai vus! - Regarde, mais regarde, c'est faux ! N'importe qui pourrait faire ça sur n'importe quel ordinateur, ok? Et j'y étais pas, donc je peux pas commenter ces images ni spéculer.»

\section{RÉSUMÉS}

Cet article aborde la question de l'illusion photoréaliste et de la production idéologique des films sous l'angle à la fois concret et circonscrit des images de synthèse des blockbusters américains. En problématisant la question du discours des films dans une perspective marxiste, j'interroge d'abord les présupposés historiques, théoriques et techniques du photoréalisme. J'examine ensuite la manière dont les images de synthèse font usage de ce dernier pour façonner, dans les films, un discours invisible, qui est généralement celui de l'American way of life, et garantir l'adhésion des spectateurs à ce dernier. Je montre que les mécanismes de la production idéologique en jeu dans les digital effects forment un système qui vise tout d'abord à dissimuler leur nature discursive, puis à oblitérer les antagonismes du réel et à assigner aux spectateurs une position de regard précise dans un ordre concret, symbolique et politique donné. 
This paper discusses the issues of photorealistic illusion and ideological production in American blockbusters through an analysis of their digital effects and computer generated images (CGI). I adopt a Marxist perspective to question their discourse. Attention is first paid to the historical, theoretical and technical conditions of the photorealism of digital effects and CGI. I then examine how films use photorealism to ensure the audience's adherence to the ideology of the American way of life. I show that the mechanisms of ideological production at play in digital effects and CGI form a system that aims first and foremost to conceal their discursive nature, then obliterate the contradictions of reality in order to assign a specific viewing position for the audience within the symbolic and political order.

INDEX

Thèmes : Hors-thème

Mots-clés : Hollywood, blockbuster, images de synthèse, idéologie, photoréalisme, American way of life, ordre politique

Keywords : Hollywood, blockbuster, digital effects, computer generated images (CGI), ideology, photorealism, American way of life, political order

\section{AUTEUR}

\section{MATHIAS KUSNIERZ}

Université Paris Diderot 